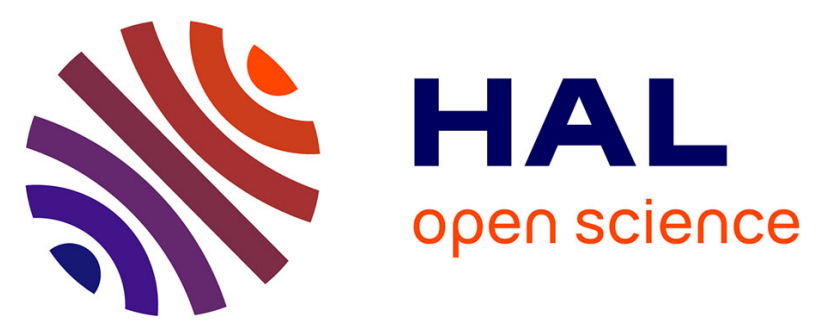

\title{
Properties of polymer/clay interphase in nanoparticles synthesized through in-situ polymerization processes
} Jenny Faucheu, Catherine Gauthier, Laurent Chazeau, Jean-Yves Cavaille, Veronique Mellon, Francis Pardal, Elodie Bourgeat Lami

\section{- To cite this version:}

Jenny Faucheu, Catherine Gauthier, Laurent Chazeau, Jean-Yves Cavaille, Veronique Mellon, et al.. Properties of polymer/clay interphase in nanoparticles synthesized through in-situ polymerization processes. Polymer, 2010, 51 (20), pp.4462-4471. 10.1016/j.polymer.2010.07.028 . hal-00539571

\author{
HAL Id: hal-00539571 \\ https://hal.science/hal-00539571
}

Submitted on 24 Nov 2010

HAL is a multi-disciplinary open access archive for the deposit and dissemination of scientific research documents, whether they are published or not. The documents may come from teaching and research institutions in France or abroad, or from public or private research centers.
L'archive ouverte pluridisciplinaire HAL, est destinée au dépôt et à la diffusion de documents scientifiques de niveau recherche, publiés ou non, émanant des établissements d'enseignement et de recherche français ou étrangers, des laboratoires publics ou privés. 


\section{Elsevier Editorial System(tm) for Polymer \\ Manuscript Draft}

\section{Manuscript Number:}

Title: Miniemulsion polymerization for synthesis of structured clay/polymer nanocomposites: Short review and recent advances

Article Type: Feature Article

Section/Category: Physics of Polymers

Keywords: Miniemulsion; nanocomposites; clay

Corresponding Author: Dr. Jenny Faucheu,

Corresponding Author's Institution: INSA-Lyon

First Author: Jenny Faucheu

Order of Authors: Jenny Faucheu; Catherine Gauthier; Laurent Chazeau; Jean-Yves Cavaillé; Véronique Mellon; Elodie Bourgeat-Lami

Abstract: This review intends to present different aspects concerning clay/polymer nanocomposites produced by heterophase polymerization in aqueous media. This paper highlights the ability of miniemulsion polymerization to produce clay/polymer latexes with tailored nanostructures. Indeed, this polymerization route enables the synthesis of composite latexes with the clay platelets located either on the surface of the latex particle or embedded inside the latex particle. A focus is given on the influence of these nanostructures on the properties of the final material through a direct comparison of the composites obtained after water evaporation from these nanostructured latexes. Indeed, the film obtained from the film forming process of these latexes present significantly different nanostructures and exhibit totally different mechanical behaviours and water uptakes. By comparing experimental results and modeling approaches, it is demonstrated that their properties are clearly related to the clay dispersion and contacts. It is also evidenced that the main drawback of miniemulsion from the coating application point of view, is the large water uptake promoted by the surfactant presence whose localization is strongly influenced by nanostructuration. This stresses the need for the further development of surfactant free miniemulsion polymerization using advantageously the clay presence to stabilize the emulsion. 
Jenny Faucheu

Saint-Etienne, October 5, 2009

Ecoles des Mines

158 cours Fauriel

42023 Saint-Etienne

faucheu@emse.fr

Dear Pr. Krausch,

As we discussed it in previous emails, you will find here the manuscript entitled «Miniemulsion polymerization for synthesis of structured clay/polymer nanocomposites: Short review and recent advances» that I modified in order to make it more suitable for publication as a feature article in Polymer.

I am the corresponding author (Jenny Faucheu) and my co-authors are Catherine Gauthier, Laurent Chazeau, Jean-Yves Cavaillé, Véronique Mellon and Elodie Bourgeat-Lami.

In particular, I added biographies of each co-authors after the paper references.

The intention of the paper remain the same that is a short review of heterophase polymerization in the presence of fillers that illustrates the challenge of producing tailored nanocomposites through direct polymerization and so highlights the breakthrough advances proposed by the use of miniemulsion polymerization for this purpose.

A second part of the paper is dedicated to personal scientific results that were obtained in the framework of a European Integrated Project, that explore this polymerization route in order to produce two series of latexes with same composition and radically different morphologies. Their direct comparison is explored to demonstrate the impact of nanostructure on properties of the final nanocomposite materials.

Hoping that this paper will suit your interest, I look forward to hearing from you.

Sincerely yours, Jenny Faucheu 

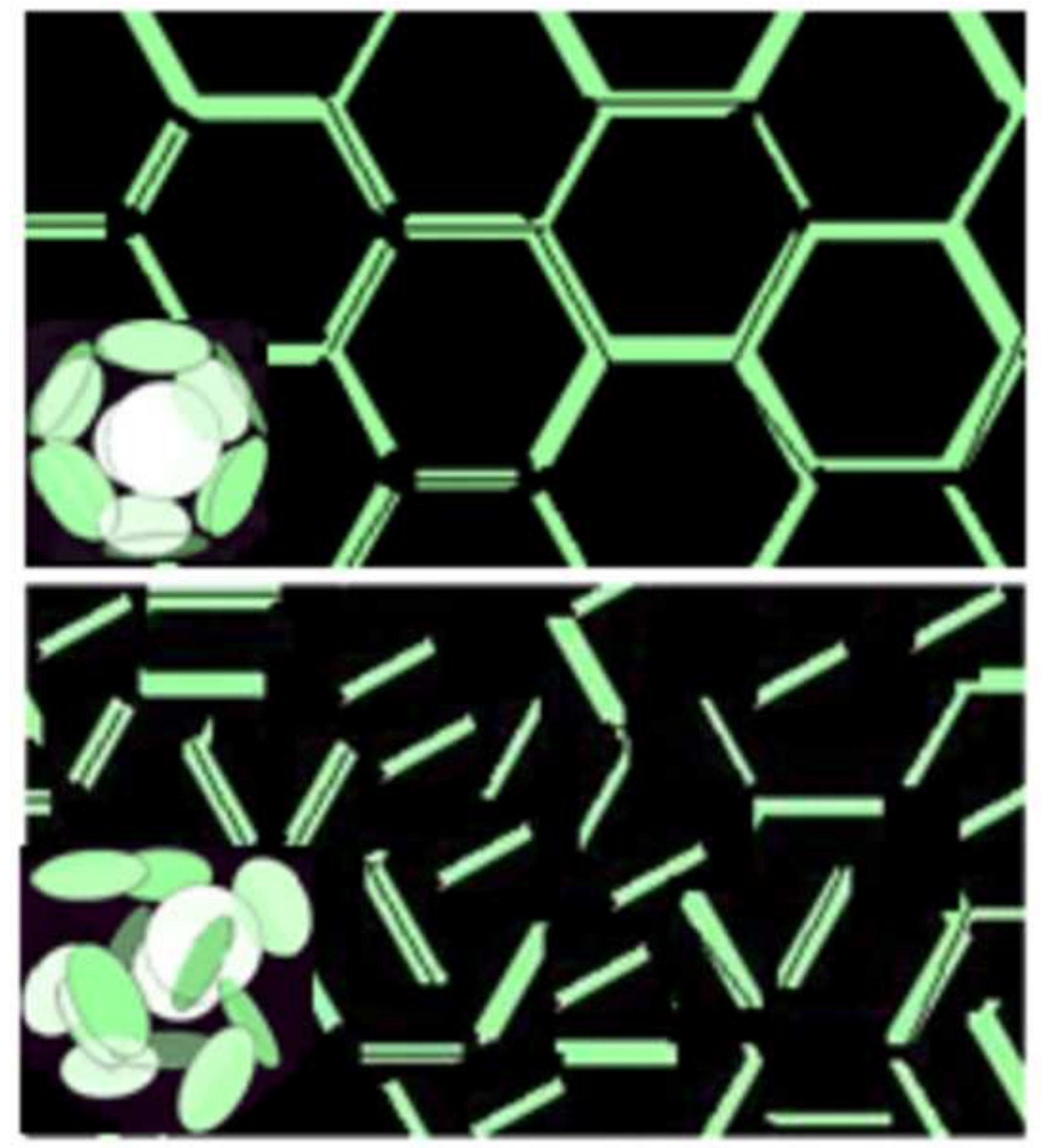


\section{Miniemulsion polymerization for synthesis of structured clay/polymer nanocomposites: Short review and recent advances}

Jenny Faucheu ${ }^{\circ *}$, Catherine Gauthier ${ }^{\circ}$, Laurent Chazeau ${ }^{\circ}$, Jean-Yves Cavaillé ${ }^{\circ}$, Véronique Mellon ${ }^{\#}$ Elodie Bourgeat Lami ${ }^{\#}$

${ }^{\circ}$ Université de Lyon, INSA-Lyon, MATEIS, 7 avenue Jean Capelle, 69621 Villeurbanne, France

\# Université de Lyon, Univ. Lyon 1, CPE Lyon, CNRS UMR 5265, Laboratoire de Chimie, Catalyse, Polymères et Procédés (C2P2), LCPP group, 43 Boulevard du 11 Novembre 1918, F-69616, Villeurbanne, France

*corresponding author: email: faucheu@emse.fr, tel: + 33477499 768, fax: +3347 7426678 


\begin{abstract}
This review intends to present different aspects concerning clay/polymer nanocomposites produced by heterophase polymerization in aqueous media. This paper highlights the ability of miniemulsion polymerization to produce clay/polymer latexes with tailored nanostructures. Indeed, this polymerization route enables the synthesis of composite latexes with the clay platelets located either on the surface of the latex particle or embedded inside the latex particle. A focus is given on the influence of these nanostructures on the properties of the final material through a direct comparison of the composites obtained after water evaporation from these nanostructured latexes. Indeed, the film obtained from the film forming process of these latexes present significantly different nanostructures and exhibit totally different mechanical behaviours and water uptakes. By comparing experimental results and modeling approaches, it is demonstrated that their properties are clearly related to the clay dispersion and contacts. It is also evidenced that the main drawback of miniemulsion from the coating application point of view, is the large water uptake promoted by the surfactant presence whose localization is strongly influenced by nanostructuration. This stresses the need for the further development of surfactant free miniemulsion polymerization using advantageously the clay presence to stabilize the emulsion.
\end{abstract}

\title{
Keywords:
}

Miniemulsion; nanocomposites; clay

\section{Introduction}

The use of particulate materials (fillers) for enhancement of polymer properties dates back to the earliest years of the polymer industry. Initially used as extending agents to reduce the cost of polymer-based products, fillers were soon recognized to be an integral component in many applications involving polymers, particularly for reinforcement purposes. Since the reinforcement efficiency of inorganic fillers is strongly related to their aspect ratio, plateletlike fillers have drawn special interest [1]. Among the different platelet like fillers, most of the published works have focused on clays.

Indeed, clay/polymer nanocomposites have been the topic of many research for decades since the results exposed by Toyota research team [2] about a montmorillonite (MMT) filled Nylon 6 after treatment with amino acids. MMT is nowadays the most widely used clay as a nanofiller. Numerous studies on nanocomposites elaboration and characterization have been 
done with all polymer families such as elastomers, thermoplastics or thermosets [3-5]. Several elaboration routes have been considered where either the nanocomposite is created from the polymer or the nanocomposite is created directly from the monomer. In all cases, the adequate surface preparation of the clays is crucial to favor their intercalation and exfoliation inside the polymer. The solvent route consists in the polymer dissolution in organic solvent and addition of the clay prior to solvent evaporation leading to polymer/clay precipitation[6-8]. But, this technique has shown difficulties in controlling the clay dispersion quality and so is much less investigated and used than the melt-compounding in both academia and industry. Indeed, the oldest and most conventional processing route is by far melt compounding [9] which is based on a direct dispersion of clays into the polymer during the melt. This processing route is very well adapted for producing high performance parts (automotive for instance) by injection molding or extrusion. However, despite the better understanding of clay/polymer interactions and the improvements in clay surface treatments, the quality of filler dispersion remains highly dependent on the mixing device. Finally, in situ-polymerization is more versatile due to variety of polymerization conditions and clay treatments and thus is a promising way to produce tailored nanocomposites with controlled morphology. Among them, heterophase polymerization in aqueous media is preferred for environmental considerations. The obtained product is a polymer latex of particles stabilized in the water phase where the filler can be either embedded or not in the particles. To control this aspect, the optimization of such polymerization route is under active research. A continuous nanocomposite film is then obtained after water evaporation and latex particles coalescence [10-13].

Actually, previous studies [14] on film formation from these polymer latexes highlighted that particles of diameter of $100 \mathrm{~nm}$ and less presented better film formation characteristics than micron scale particles. Thus, researchers have integrated this size parameter in their product requirements. This reduced the choice of clays with adequate size characteristics that can be incorporated within nanoscale polymer latex particles. Among potential candidates, Laponite, a disk shape synthetic smectite clay $(25 \mathrm{~nm}$ in diameter and $0.92 \mathrm{~nm}$ thick) has attracted particular attention. Indeed, Laponite has not only small dimensions compared to MMT, but also offers the advantage of being free of chemical impurities and of constant quality. Specific surface areas ranging from 370 to $900 \mathrm{~m}^{2} \cdot \mathrm{g}^{-1}$ have been reported $[15,16]$. Due to its nanometric size and its similarities with MMT, Laponite is a promising candidate for polymer/clay colloidal nanocomposite processing and a serious competitor to MMT. 
Finally, considering environmental and economical aspects, heterophase polymerization has raised an increasing interest, more particularly, heterophase polymerization in the presence of nanoclays appears as a suitable way to produce clay/polymer nanocomposites. Emulsion polymerization and more recently, miniemulsion polymerization are both used for naocomposite synthesis.

Hereafter, in a first part, several aspects concerning clay/polymer nanocomposites produced by such techniques are reviewed, with a special emphasis on miniemulsion polymerization, which enables to obtain clay-armored latex particles similarly to emulsion polymerization but also latex particles with encapsulated clays.

In a second part, in-situ miniemulsion polymerization is effectively adopted to produce two nanocomposite materials with distinct morphologies. Indeed, motivated by such advantage of the miniemulsion polymerisation, a European research program has brought together several research teams with the common goal of producing Laponite/polymer nanocomposite latexes with controlled morphologies via this technique. In this framework, two polymer/clay morphologies were obtained. In a first series of latexes, the Laponite platelets were covering the surface of the latex particles giving rise to a clay network architecture after film forming process. In a second series of latexes, in contrast, the platelets were encapsulated inside the latex particles resulting in inorganic particles uniformly dispersed within the film material. Therefore, the second part of this paper is dedicated to the synthesis and the characterization of these new materials in order to address the impact of clay topological distribution on the final film properties.

\section{Heterophase polymerization for synthesis of nanocomposite}

From a general point of view, polymerization techniques can be divided into two types of chemical reactions: step-growth polymerization and free radical polymerization. Step-growth polymerization is widely used for synthesis of polyesters, polyamide and epoxies while the synthesis of polyacrylics requires the use of free radical polymerization. These polymerization reactions can be performed either in bulk or in solution or in dispersed media. Heterophase polymerizations (i.e. emulsion, dispersion and miniemulsion polymerizations) present the advantage of easier removal of the resulting product from the reactor compared to bulk polymerization thanks to the low viscosity of the reaction medium. Polymerization in solution also induces lower viscosity but also lower reaction rates due to dilution of the reactants and higher cost and environmental impact due to the use of organic solvents. These problems are solved in the case of heterophase polymerizations where the reactants are confined inside 
droplets (no dilution effect) and water is used as medium. The use of surfactant molecules are usually needed for the stabilization of the monomer droplet and subsequent polymer particles in the water phase.

To our knowledge, the first composite synthesis through emulsion polymerization has been reported in 1993 by Lee et al. [17]. The emulsion polymerization mechanism is divided in two main stages, first the nucleation (in water phase or surfactant micelles) and the particle growth where monomer droplets act as reservoirs to feed the reaction with diffusion of monomer molecules through the water phase to the reaction loci. The authors described the synthesis of PMMA latex particles in the presence of $\mathrm{Na}^{+}-\mathrm{MMT}$ without any coupling agents and using a water soluble initiator. The obtained products were exposed to boiling toluene for 5 days in order to extract the non-bonded polymer, which was further analyzed in terms of molecular weight $(\mathrm{Mw})$. It appeared that the Mw of extracted PMMA was comparable to that of pure PMMA. Since this pioneer work, there has been increasing interest for in-situ emulsion polymerization. Noh et al. [18] highlighted the advantages of emulsion polymerization over solution polymerization by comparing poly(styrene-acrylonitrile)/MMT (SAN/MMT) nanocomposite materials similar in composition but obtained from each synthetic route. An unmodified MMT was used for emulsion polymerization whereas an organically-modified MMT was used for solution polymerization. Additional microstructural characterizations highlighted the irregular dispersion of relatively large-sized MMT stacks in the solutionsamples. Noh et al. concluded that emulsion polymerization in the presence of clays should be a promising route to produce polymer/clay hybrid nanocomposites. Considering that these first in-situ emulsion polymerizations were performed with pristine MMT without specific surface modification, it is worth noting that tremendous improvements were expected from an organic modification of the clay surface that would render it compatible with monomer and polymer and/or even reactive during polymerization.

Three major categories of clay modifications have been reported: cation exchange, silane grafting and adsorption of polar polymers. Cation exchange is based on the exchange of the structural inorganic cations of the clay interlayer by an organic cation that can bring in some cases a reactive group. For instance, Fan et al. [19] reported the synthesis of a cationic free radical initiator and its intercalation into MMT galleries to initiate the free radical polymerization of styrene from the clay surface. The amount of organic cations that can be exchanged depends on the amount of exchangeable sites and therefore depends on the cation 
exchange capacity (CEC) and the structure of the phyllosilicate. On the other hand, organosilanes are molecules with the general formula $\mathrm{R}_{\mathrm{n}} \mathrm{SiX}_{4-\mathrm{n}}$, where $\mathrm{X}$ is an hydrolysable group (halogen, alkoxy, etc.) and $\mathrm{R}$ represents a non hydrolysable organic moiety that can form covalent bonds with clay surfaces. Indeed the broken edges of the clay sheets offer the presence of hydroxyl groups that can react with organosilanes to form siloxane bonds. Then, by choosing appropriate organosilane molecules carrying a polymerizable moiety, a covalent bond can be created between the silicate filler and the polymer matrix. Although numerous studies have been reported on the grafting of silane coupling agents to the surface of mineral oxide particles, comparatively only a limited number of works have been published on the silylation reaction of clays [20].

As it was mentioned previously, MMT is the most studied clay filler, and due to its similarities with Laponite clays, most of these surface treatments have also been performed on Laponite. The crystal structure of Laponite consists of two dimensional layers with a magnesium-oxygen octahedral sheet sandwiched between two silicon-oxygen tetrahedral sheets. Isomorphic substitution of some octahedral magnesium ions by lithium ions in the crystal structure generates negative charges that are compensated by the presence of sodium cations in the interlayer space. The Laponite CEC was reported to be $75 \mathrm{meq} / 100 \mathrm{~g}$ [21]. In addition to interlayer charges, uncoordinated silicon or magnesium ions located at the broken edges of the crystal sheets react with water to form hydroxyl groups. These groups are amphoteric and may be negatively or positively charged depending on the $\mathrm{pH}$ of the dispersion. Interactions between the positively-charged rims and the negatively-charged faces are responsible for the formation of gels with the so-called house-of-cards architecture. A peptizing agent (e.g. tetrasodium pyrophosphate) can be added to Laponite suspensions to hinder gel formation. This tetravalent negatively charged ion adsorbs on the rim of the Laponite platelets and thereby screens the face-to-rim interactions. Negrete Herrera et al. [22] reported the grafting of $\gamma$-methacryloxy propyl trimethoxysilane on the edges of Laponite clay platelets. The organoclays were used as seeds to synthesize poly(styrene-co-butyl acrylate)/Laponite nanocomposite latexes through emulsion polymerization. Finally, taking advantage of the fact that some polar polymers spontaneously adsorb on clay surfaces [2325], a methacrylate-terminated PEG-based macromonomer was recently used to functionalize the surface of Laponite clay platelets which were engaged further in soap-free emulsion polymerization. Negrete-Herrera et al. [21] also reported the synthesis of clay-armored latex particles using organically-modified Laponite clay platelets via emulsion polymerization. The 
Laponite platelets were modified through cation exchange with either (2,2-azobis(2methylpropionamidine) hydrochloride (AIBA) used as polymerization initiator or 2(methacryloyoxy) ethyl trimethyl ammonium chloride (MADQUAT) used as comonomer. The modified clays were dispersed in the water phase prior to emulsification with the monomer phase.

Similar clay-armored latex morphology was also obtained using another polymerization route so-called miniemulsion polymerization. This heterophase polymerization differs from conventional emulsion polymerization. Miniemulsion is ideally described as a one-to-one mechanism meaning that one monomer droplet leads to one latex particle. This specific mechanism is favored by the addition of a co-stabilizer (or hydrophobe) that hinders Ostwald ripening and diffusion of monomer from a droplet to another. A detailed description of miniemulsion polymerization mechanism has been reviewed by Schork et al. [26]. In miniemulsion polymerization, a special attention is given to the generation of a small emulsion droplets [27] in order to favor the nucleation of the nanodroplets and avoid homogeneous and micellar nucleation (predominant in emulsion polymerization). This new synthetic route seems promising for a fine design of the morphology of nanocomposite latex particles and increasing work is devoted to it. For instance, Laponite armored-latex particles of polystyrene made via a Pickering stabilized miniemulsion polymerization was synthesized by Cauvin et al. [28]. Tong et al. [29] reported that a polystyrene nano-saponite composite suspension was synthesized via miniemulsion polymerization. They found that the addition of clay slightly reduced both the reaction rate and the conversion. Moraes et al. [30] produced poly(styrene-co-butyl acrylate)/ montmorillonite via miniemulsion polymerization. The natural clay was previously treated with Cetyltrimethyl ammonium chloride (CTAC) in order to increase the interaction between the clay and the monomer phase. They reported no influence of the introduction of organically modified clays on the kinetics of polymerization.

The real breakthrough advantage of miniemulsion over conventional emulsion polymerization is the fact that clays can be effectively encapsulated inside the polymer particles. Indeed, taking advantage of the fact that the latex particles are the replica of the miniemulsion droplets, it is possible to encapsulate the clay platelets provided that the later are initially dispersed inside of the monomer droplets. This needs the functionalization of the clays with an organophilic treatment to render them compatible with the monomer phase. In 2006, Sun et al. [31] first reported the encapsulation of Laponite platelets in polystyrene latex particles via 
miniemulsion polymerization. The Laponite platelets were modified through cation exchange with cetyltrimethylammonium bromide $(\mathrm{CTAB})$ and mixed in the monomer phase prior to emulsification with water. In 2008, Samakande et al. [32] reported the successful encapsulation of MMT in polystyrene latex particles by Reversible Addition-Fragmentation Chain Transfer (RAFT) mediated miniemulsion polymerization. Raft grafted MMT were dispersed in styrene monomer and emulsified in the presence of a hydrophobe (hexadecane) into miniemulsions that were polymerized leading to encapsulated polystyrene-clay nanocomposites.

This review on nanocomposites synthesized by in-situ polymerization highlights an increasing number of works on emulsion polymerization. Compared to bulk or solution polymerizations, this technique limits the problem of clay agglomeration in the polymer matrix formed after evaporating the latex suspension. Moreover, there is no problem of increased viscosity at the end of polymerization like in bulk polymerization and this technique does not suffer of drawbacks of dilution (lower kinetics) like in the case of solution polymerization. For now, a lot of works have focused on the clay dispersion quality and take advantage of the advances in clay surface treatments. However, it appears that emulsion polymerization is not adequate for clay encapsulation where the obtained nanostructure mostly consists of clays covering the surface of latex particles. The recently developed miniemulsion polymerization enables to fill this gap and to synthesize latex particles with tailored morphologies: the clay platelets either covering the surface of the latex particles as previously or being embedded inside the latex particle.

\section{3. miniemulsion polymerization for synthesis of tailored structured clay/polymer nanocomposites}

Motivated by the advantages of miniemulsion polymerization, this technique has been explored in a European project to produce polymer/Laponite composite latexes of controlled nanostructure which were ultimately processed into films. Two nanostructures were synthesized. Clay-armored latex particles were first obtained through miniemulsion polymerization by anchoring a free initiator to the clay surface through cation exchange. This treatment renders the clay particles amphiphilic and promotes subsequent clay interaction with the miniemulsion droplets. The clay with attached radicals nucleates the nanodroplets that progressively form particles consisting of an outer shell of clay sheets surrounding an inner polymer core. On the other hand, encapsulated clay particle were synthesized thanks to 
an appropriate clay treatment as detailed further. All polymerizations were performed in a $500 \mathrm{~mL}$ four-neck reactor equipped with a nitrogen inlet-outlet tube, a condenser and a mechanical stirrer. Laponite (RD grade) was supplied by Laporte Industries. The monomers, methyl methacrylate and butyl acrylate (from Aldrich), were used as received. The composition of the acrylic copolymer is 50/50. Others components of the recipe are the initiator (azobis(isobutyramide di-hydrochloride) AIBA) or 4'-azobis (4-cyanopentanoic acid) (ACPA) and the hydrophobe (Behenyl acrylate (BylA) Norsocryl from Arkema). All latexes were prepared at $20 \mathrm{wt} . \%$ solid content. A conversion around $80 \%$ was reached for all reactions.

\subsection{Clay-armored latex particles (C-series)}

For the first series, a cationic initiator (AIBA) was incorporated in the Laponite layers through cation exchange. This specific functionalization of the Laponite basal faces renders the clay platelets reactive and ensures the formation of the hybrid nanostructure by initiating the polymerization at the surface of the platelets [22]. The cation exchange was carried out at $200 \%$ of the cation exchange capacity (CEC) of Laponite reported at 75meq/100g [22]. Note that a sample without Laponite (C0\%) is synthesized with $0.4 \mathrm{wt} \%$ of AIBA. The AIBAintercalated Laponite platelets were next dispersed in water in the presence of tetrasodium pyrophosphate (peptizer content is based on laponite content, given in Table 2) and Dowfax (surfactant, $2 \mathrm{wt} \%$ active compound based on monomers, given in Table 2). The monomers and Norsocryl (hydrophobe, $6 \mathrm{wt} \%$ based on monomer content) were then mixed and poured in the clay suspension under magnetic stirring. The resulting emulsion was sheared further by sonication with a Branson sonicator for $15 \mathrm{~min}$. The polymerization was carried out at $70^{\circ} \mathrm{C}$ for 3 hours under nitrogen flow. As the Laponite carries the initiator, the polymerization starts from the clay surface. Moreover, due to the fact that the Laponite rims remain unmodified, these clays might be both hydrophilic and hydrophobic and are thus expected to be located at the monomer/water interface and therefore on the surface of the final latex particles. Latexes with three different laponite contents have been synthesized; the laponite content has been measured by thermogravimetric analysis and is reported in Table 1. These samples are called C-series (Cellular) due to the nanostructure of the material obtained after film forming process. Indeed, the nanocomposites exhibit a cellular morphology with cells of the size of the mother latex particles and walls made of clay platelets as depicted in the TEM micrograph (figure 1a). Due to their very small thickness, the Laponite platelets are visible when they are edge up, then they appear as dark sticks on a TEM micrograph. If they are face up, single 
platelets do not have enough contrast with the polymer background. The image of $\mathrm{C} 10 \%$ film is presented in Figure 1a: dark contours (Laponite platelets) are visible outlining grey areas (polymer matrix). This comforted the nanostructure expected from the reaction mechanism (depicted above): the Laponite platelets form a cellular structure throughout the film, meaning that they are located on the surface of the mother latex particle.

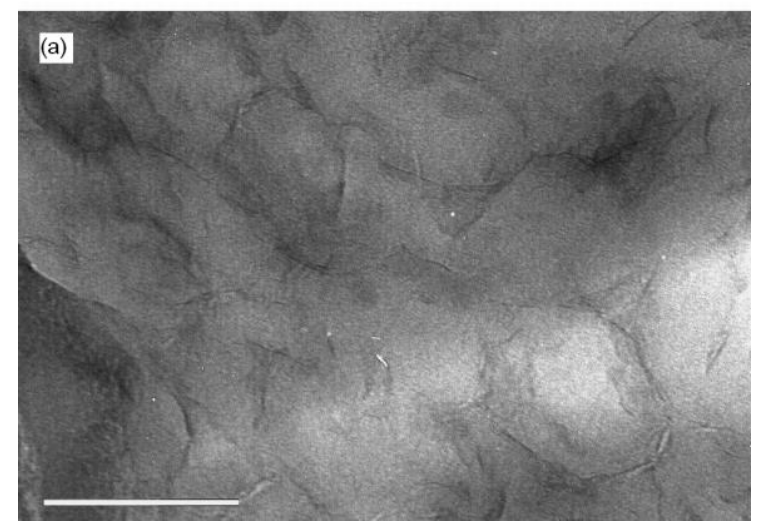

Figure 1a: TEM image of sample C10\%, Thin films obtained by evaporating a drop of diluted latex on a carbon coated grid. (JEOL 200CX, accelerating voltage 200keV). scale bar 200nm.

\subsection{Encapsulated-clays latex particles (D-series)}

An organosilane coupling agent, $\gamma$-methacryloxypropyltrimethoxysilane ( $\gamma$-MPTMS) was grafted to the Laponite rims (25wt\% based on laponite content, Table 2) following a procedure reported in the literature [33]. In addition, a quaternary alkyl ammonium surfactant, didodecyldimethylammonium bromide (DDAB) ( $8 \mathrm{wt} \%$ based on laponite content, Table 2), was intercalated in the Laponite layers through cation exchange. Note that a sample without Laponite (D0\%) is synthesized without $\gamma$-MPTMS and DDAB. This double modification procedure (basal faces and clay edges) was performed in order to ensure a good compatibility of the clay with the MMA/BA monomer mixture. The DDAB- $\gamma$ MPTMS-intercalated Laponite platelets were dispersed in the monomers with Dowfax (surfactant, $2 \mathrm{wt} \%$ active compound based on monomers, given in Table 2) and Norsocryl (hydrophobe, 6wt\% based on monomer content) under magnetic stirring for 12 hours followed by 3 min sonication. Water was added to the resulting dispersion under magnetic stirring. The resulting emulsion was sheared further by sonication with a Branson sonicator for $15 \mathrm{~min}$. The polymerization was started by the injection of ACPA ( $0.4 \mathrm{wt} \%$ based on organic content) and was conducted at $70^{\circ} \mathrm{C}$ for 3 hours 
under nitrogen flow. As the Laponite platelets present a good compatibility with the monomers, they are expected to remain inside the monomer droplets and be therefore inside the latex particles. After film formation, the nanocomposite should exhibit a good dispersion of clays without any specific ordering.

These samples have been referenced as D-series (Dispersed) and their laponite content are given in Table 1. The TEM micrograph of the D5wt\% latex is different (Figure 1b): in that case, round objects with a darker core were observed, which evidences that Laponite are more likely confined inside the particle. However it seemed that some platelets might also be located on the surface of the particles. Similar TEM images were presented by Sun et Al. [31] for polystyrene latex with encapsulated Laponite.

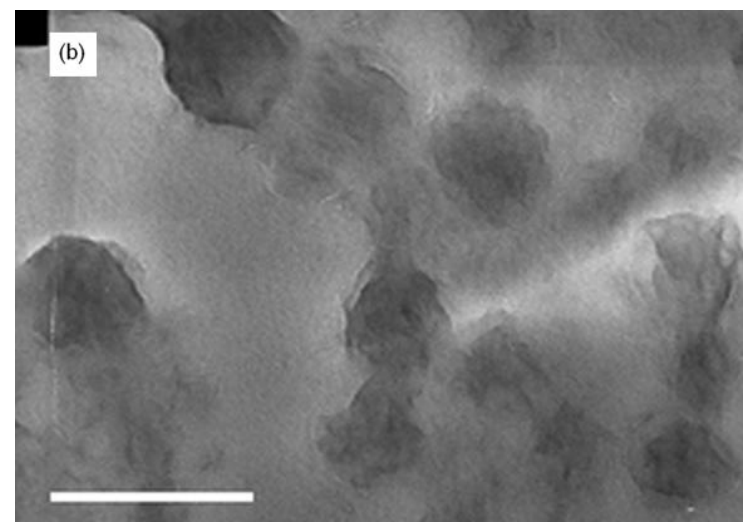

Figure 1b: TEM image of sample D5\%, Thin films obtained by evaporating a drop of diluted latex on a carbon coated grid. (JEOL 200CX, accelerating voltage 200keV). scale bar 200nm.

Table 1: sample references obtained by miniemulsion polymerizations of MMA/BA (50/50) for the $\mathrm{C}$-series and the $\mathrm{D}$-series.

\begin{tabular}{lll|lll}
\hline Sample & $\begin{array}{l}\text { Laponite } \\
(\mathrm{wt} \%)^{\mathrm{a}}\end{array}$ & Particle ${ }^{\mathrm{b}}$ size (nm) & sample & $\begin{array}{l}\text { Laponite } \\
(\mathrm{wt} \%)^{\mathrm{a}}\end{array}$ & Particle ${ }^{\mathrm{b}}$ size (nm) \\
\hline $\mathrm{C} \mathrm{0 \%}$ & 0.0 & 68 & D 0\% & 0.0 & 97 \\
$\mathrm{C} \mathrm{6 \%}$ & 5.7 & 97 & D 3\% & 3.4 & 100 \\
$\mathrm{C} 8 \%$ & 8.1 & 83 & D 5\% & 5.1 & 110 \\
$\mathrm{C} \mathrm{10 \%}$ & 10.0 & 81 & D 7\% & 6.5 & 117 \\
\hline
\end{tabular}

${ }^{a}$ Laponite content measured by thermo-gravimetric analysis (ATG) using NETSZCH T209 $\left(30^{\circ} \mathrm{C}\right.$ to $800^{\circ} \mathrm{C}$ under air flow). ${ }^{b}$ Determined by Dynamic Light Scattering (DLS) using a Malvern Autosizer Lo-C.

In summary, the two synthetic routes we adopted in this work enable to produce well-defined nanostructured polymer/Laponite latexes with radically different morphological characteristics, as depicted in figure 2. Both latex series present a particle size around 100nm 
diameter, however, C-series present slightly smaller particles (ca. 85nm) compared to Dseries (ca. 105nm). All the latexes were able to film form at room temperature as requested for a coating application.

\section{Low clay content}

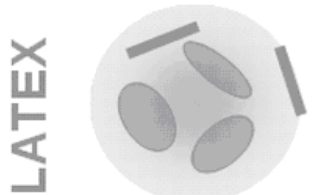

D-series
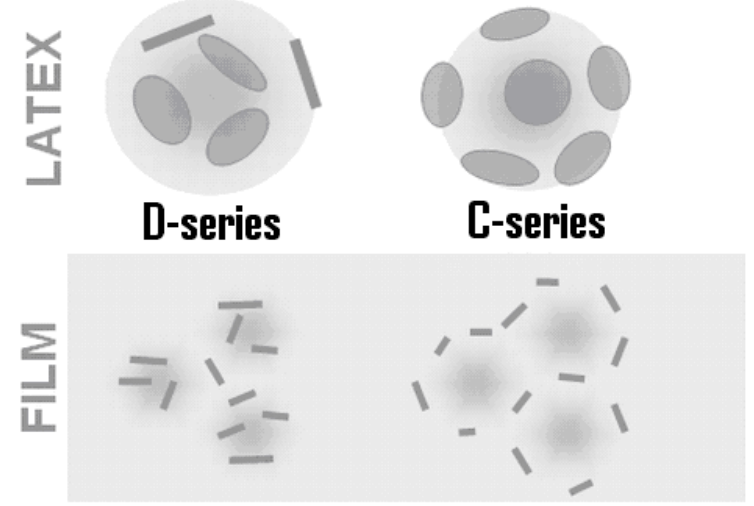

C-series
High clay content

D-series

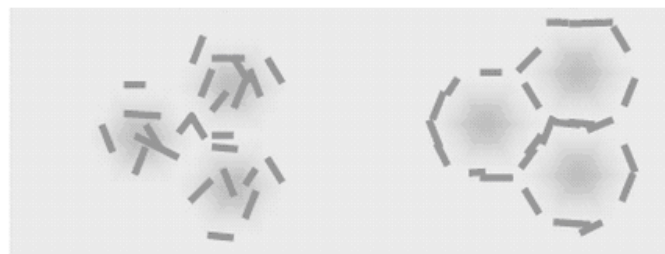

Figure 2: Scheme of the latex particle nanostructure and the resulting composite film for "low" and "high" Laponite contents.

\subsection{Quality of clay dispersion within the polymer matrix for both $C$-series and D-series}

Using SAXS experiment, the basal plane spacing of pure laponite was found equal to $1.39 \mathrm{~nm}$ $\left(q=4.51 \mathrm{~nm}^{-1}\right)$. The same value was reported by Negrete-Herrera et al. [21]. The scattering intensity I(q) for C6\% and D5\% samples is plotted in Figure 3, the upper curves have been shifted for clarity. Both present a peak for q values in the 1 to $10 \mathrm{~nm}^{-1}$ range. This means that both nanocomposites contain intercalated clays with larger basal plane spacings $(2.82 \mathrm{~nm}$ $\left(\mathrm{q}=2.22 \mathrm{~nm}^{-1}\right)$ for $\mathrm{C} 6 \%$ sample and $3.06 \mathrm{~nm}\left(\mathrm{q}=2.53 \mathrm{~nm}^{-1}\right)$ for D5\% sample. Thus, these nanocomposites might contain exfoliated clays (that do not scatter X-Rays in this q range) and in addition they both contain stacks of intercalated clays with an interlayer distance of ca. $3 \mathrm{~nm}$.

At smaller q values, we adopt the usual assumption that the spatial arrangement of the Laponite disks (structure factor $\mathrm{S}(\mathrm{q})$ ) is decoupled from the form factor $\overline{F^{2}}(q)$ [34]. Thus the scattered intensity is given by equation (1):

$$
I(q) \propto \rho I_{0} S(q) \overline{F^{2}}(q)
$$

with $\rho$ the number density of individual scatterers in the sample and $\mathrm{I}_{0}$ the incident intensity. $\mathrm{S}(\mathrm{q})$ accounts for the interference of radiation scattered from the particles in the structure. $\overline{F^{2}}(q)$ accounts for the interference of radiation scattered from different parts within a 
particle. Between 0.1 and $1 \mathrm{~nm}^{-1}, \mathrm{I}(\mathrm{q})$ roughly follows a $\mathrm{q}^{-2}$ power-law decay for both $\mathrm{C}$ and $\mathrm{D}$ samples, which is consistent with the form factor $\mathrm{F}(\mathrm{q})$ of randomly oriented thin disks [34, 35]. At a volume fraction of ca. $2 \mathrm{vol} \%$ (i.e. $5 \mathrm{wt} \%$ ), the condition of diluted platelets can be accepted for both samples. However, it appears that the D sample does follow a $q^{-2}$ decay whereas the $\mathrm{C}$ sample follows a $\mathrm{q}^{-2.3}$ decay. This slight difference could be due to the cellular structure in the $\mathrm{C}$ sample that degrades the randomness of the clay dispersion.

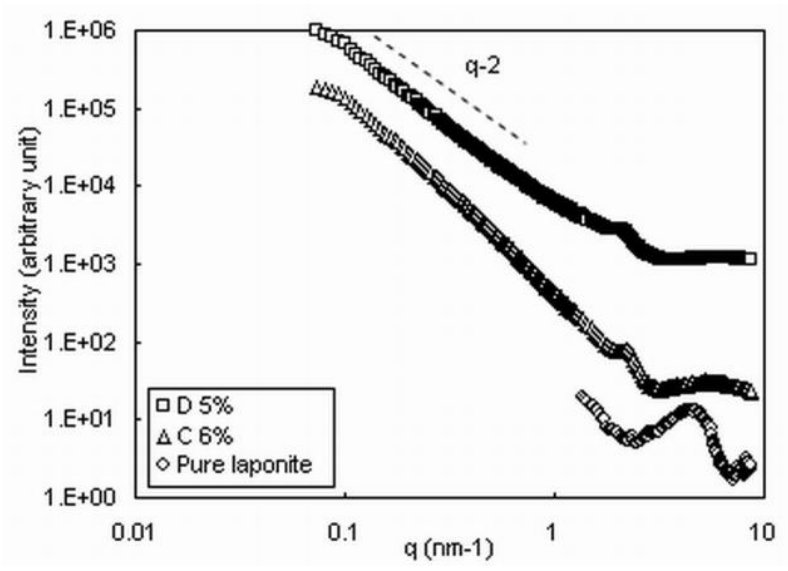

Figure 3: SAXS results for pure laponite, C6\% and D5\% samples (SAXS experiments were performed on $1 \mathrm{~mm}$ thick pile of films)

A comparison of the impact of these latex morphologies on film properties is an interesting area to be investigated. To probe the properties of the material, films were made by pouring diluted latex (10\% solid content) in a Teflon plate covered with a piece of paper to avoid moving air above the surface and placed at $23^{\circ} \mathrm{C}$ until a crack-free and transparent film was formed. The films obtained were $0.35-0.45 \mathrm{~mm}$ thick. Experimental results of the mechanical response of both series of materials are reported hereafter.

\section{Properties of tailored structured nanocomposites obtained via miniemulsion polymerization}

\subsection{Mechanical properties}

Whatever the processing route, the expected impact of addition of nanofillers in polymer matrix is an increase of the modulus, also called reinforcement. From a general point of view, the modulus increases with the filler content and the aspect ratio, however it also depends on the dispersion, the filler-to-filler and filler-to-matrix interactions. In the past, structural effects induced by process have already been reported, for instance, the processing of fiber/polymer by extrusion led to composites with highly aligned fiber. Such structured composites raised a 
keen interest due to strongly anisotropic properties that were experimentally observed. Similar anisotropic effect are also reported for platelets like fillers [36, 37]. For nanocomposites produced by polymerization in emulsion, it appears that the focus was, for now, on the feasibility of producing good clay dispersion and only few studies investigated the mechanical properties. Among the few, Lee et al. [17] reported enhanced properties for the nanocomposite products compared to the pure PMMA: for instance enhanced thermal stability and increased modulus (from 3.06GPa for the pure PMMA to 4.64GPa for a composite containing 10wt.\% MMT). Noh et al. [18] compared nanocomposites produced by solution polymerization and emulsion polymerization; they reported that while the values of modulus and elongation at maximum load were found to be comparable, the stress at maximum load of the solution-nanocomposite was found twice lower that the emulsion-nanocomposite. This was attributed to different intercalation states that impact reinforcement through the modification of the aspect ratio.

In addition to results reported in literature, the properties of the films obtained in the frame of the EU Napoleon project are discussed hereafter. Figure 4 plots the evolution of the storage modulus with temperature from $-100^{\circ} \mathrm{C}$ to $120^{\circ} \mathrm{C}$. As expected, the transition from glassy to rubbery state of all samples was located around the coating application temperature between $0^{\circ} \mathrm{C}$ and $50^{\circ} \mathrm{C}$. One can notice small evolutions of the main relaxation temperature that has been addressed in another article and attributed either to a modification of the mobility of the copolymer in the polymer-filler interphase or to a composition drift of the copolymer [38]. These shifts will be neglected in the discussion of the mechanical reinforcement that will be considered at temperature well above the main relaxation temperature i.e. when the polymer is in the rubbery state.

The behavior of the composite films at small deformations reveals a strong impact of Laponite addition in particular for the $\mathrm{C}$ nanostructure. For the $\mathrm{C}$ samples, an increase of the level of the rubbery plateau with increasing Laponite content ( 0 to $10 \mathrm{wt} \%)$ is observed, in particular, the level of rubbery plateau increases clearly between sample $\mathrm{C} 6 \%$ and $\mathrm{C} 8 \%$. For the D samples, an increase of the rubber plateau is observed for the D3\% and D5\% however the sample D7\% exhibits a response similar to sample D5\%. This response of sample D7\% might be due to a poorer clay dispersion (presence of stacks that lower the aspect ratio for instance) in comparison to sample D5\%. 

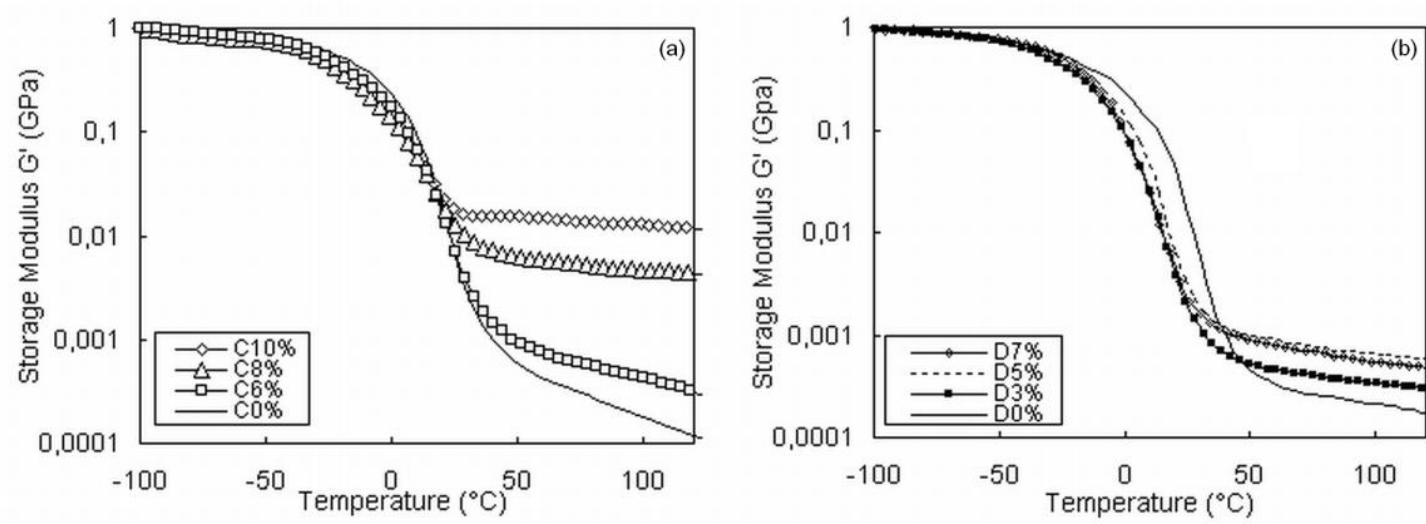

Figure 4: Storage modulus vs. temperature obtained by DMA for (a) C-series and (b) Dseries. DMA in torsion mode at 1Hz and 10-2rad angle 1K.min-1. Sample dimensions: $10 \mathrm{~mm}$ $x 3 \mathrm{~mm} \times 0.35-0.45 \mathrm{~mm}$. Glassy level normalized to $1 \mathrm{GPa}$

The large influence of the nanostructure can also be evidenced by stress-strain curves. Figure 5a shows the strain-stress plots of the $\mathrm{C}$ samples obtained from uniaxial tensile tests performed at $70^{\circ} \mathrm{C}$ (in the rubbery state). Figure $5 \mathrm{~b}$ shows the results for the $\mathrm{D}$ samples under similar experimental conditions. All samples undergo a very large elongation without breaking. Only the behavior for true strains below 0.5 has been plotted. For $\mathrm{C}$ nanostructured samples, a sharp increase of the elastic modulus (initial slope) is observed which is consistent with the previous DMA. For the D samples, a slight increase of elastic modulus is observed from the blank polymer to the samples D3\%, however no change is observed between the samples D5\% and D 7\%.
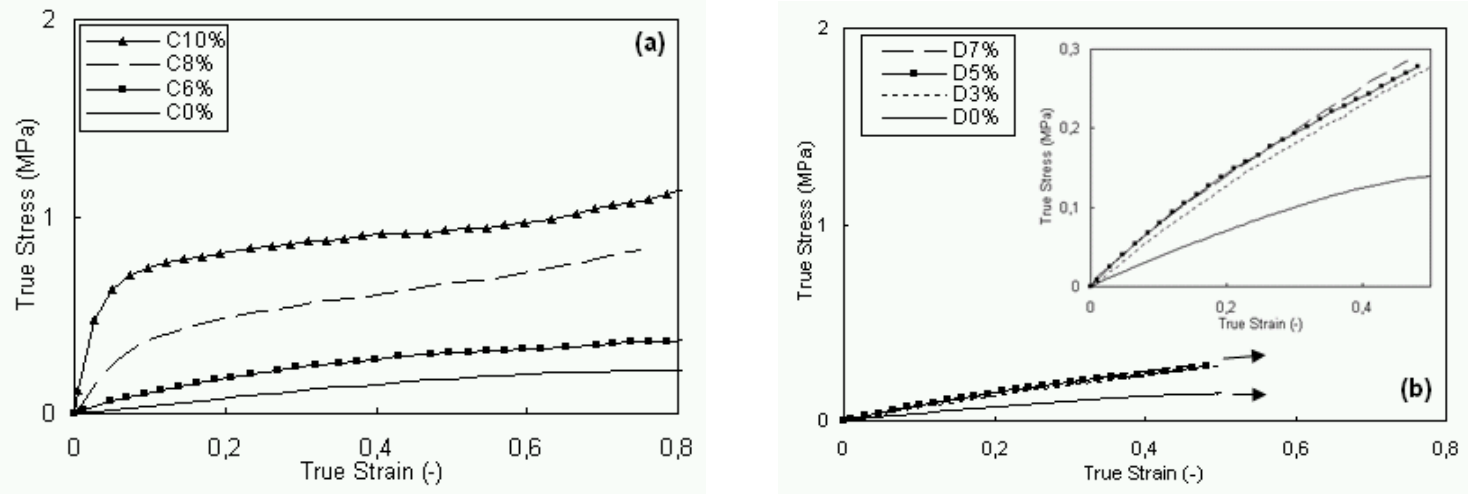

Figure 5: True stress vs. true strain for (a) C-series and (b) D-series

Uniaxial tensile test at $T \alpha+50^{\circ} \mathrm{C}$ and $\dot{\varepsilon}=0.005 s-1$ using a VideoTraction apparatus [39].

The high reinforcement level and shape of stress strain C-nanostructured clay/polymer nanocomposites is very similar to those obtained with whiskers/polymer nanocomposites. 
This suggests the presence of a percolating network of fillers, in which the filler-filler interactions are strong. Thus, it is expected that this percolating network should be destroyed under larger deformation. To highlight this damage phenomenon, cyclic tensile tests have been performed. The equipment and sample shapes were the same than for standard tensile test with VideoTraction equipment. The sample was pulled to 0.5 true strain value before being pulled back at the same rate to zero stress value. Once there, the sample was pulled again to 0.5 true strain value. This sequence was then repeated twice. The samples were tested at $\mathrm{Tg}+50^{\circ} \mathrm{C}$ at $\dot{\varepsilon}=0.005 \mathrm{~s}^{-1}$. The data were post-analyzed taking into account the deformation remaining after each cycle by correcting the dimensions of sample in the stress calculations. Figure 7 presents corrected plots corresponding to the three successive cycles. For all samples, a viscoelastic behavior is obtained characterized by the hysteresis. For the D5\% samples (Figure 6a) the elastic modulus remains the same after the first tensile cycle whereas for the $\mathrm{C} 10 \%$ sample (Figure $6 \mathrm{~b}$ ) the modulus drops after the first cycle. This showed the damage probably due to the disruption of the percolating network of Laponite, is occurring in C samples while undergoing large deformation. On the contrary, the D sample behaves similarly to pure viscoelastic polymer without any damage. These observations support the idea that the stresses are mainly transferred through the matrix for the D samples whereas they are transferred through Laponite-Laponite contacts in the $\mathrm{C}$ samples.
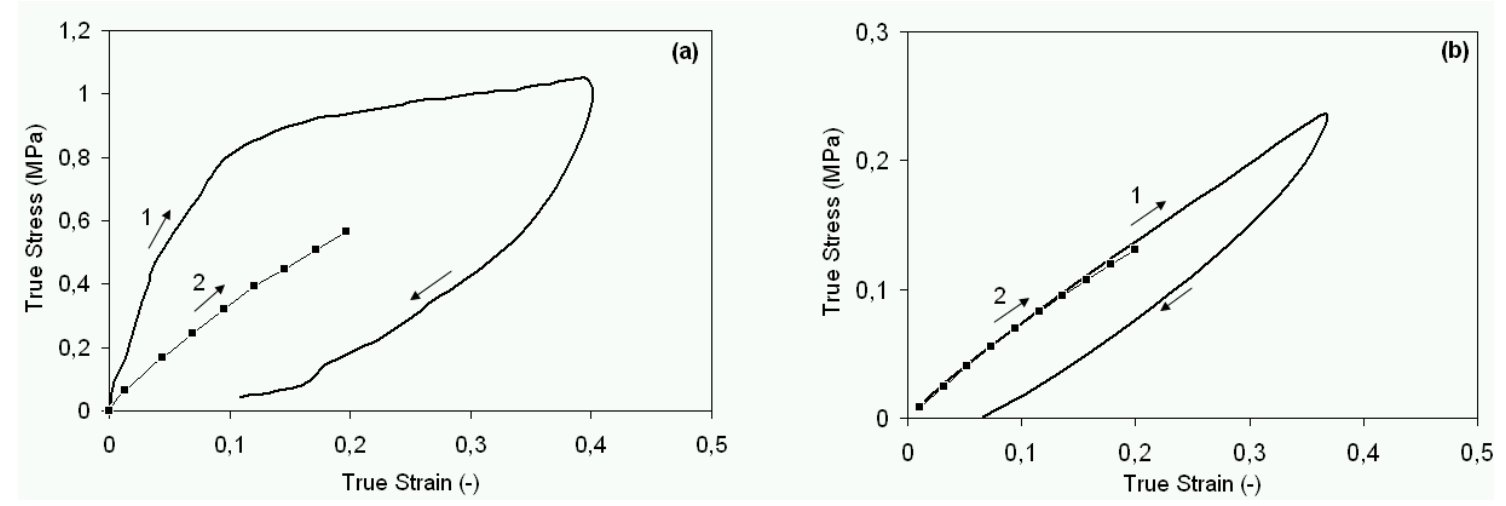

Figure 6: Cyclic tensile test of (a) C10\% sample and (b) D5\% sample.

This example confirms that the specific nanostructure that promotes strong contacts between clays leads to very high reinforcement level and to propensity to damage. This is in agreement with general results on nanocomposites mechanical properties reported in literature [1].

\subsection{Modeling of the mechanical behavior}


To go further in the analysis of the mechanical properties of nanocomposites, modeling tools are developed to reproduce the experimental data and thus to better understand the parameters that impact the properties. On the other hand, such models are also developed to propose a tool for tailored design of materials for specific application requirements. Variety of approaches has been considered to reproduce composite behavior from the intrinsic properties of the constituents from more or less handy analytical equations (e.g. Christensen and Lo [40, 41], Halpin and Tsai $[42,43]$ and Tandon and Weng [44]) to numerical and time-consuming approaches (e.g. Finite Element, FE) [45]). The first approach has the advantage of being handy to use. For instance, two phases models for prediction of elastic modulus have been reviewed by Ahmed et al.[46]. However, whereas this approach is usually adequate to predict composite behavior, recent papers report its limits in the prediction of nanocomposite behavior due to the presence of a high volume of interphase. Thus, it has been argued that a model that account for the presence of three phases (inclusion/interphase/matrix) should be more adequate than a model with only two phases (inclusion/polymer). Ji et al. [47] proposed a model based on series and parallel formulation taking into account all three phases. On the other hand, FE approach seems more adequate than analytical approches as the Representative Volume Element that is considered is supposed to closely reproduce the real materials. Such three dimensions Finite Element (FE) approach has been used to study composite materials with specific structure such as aligned fibers or random silica beads [48]. To avoid overlong calculation times, the representative volume element (RVE) must be reasonably small. A periodic RVE is often used, where the particles that are cut from any of the faces of the RVE are continued from the opposite face with the same orientation. Studying a polymer composite filled with glass spheres, Gusev [49] found good results for a RVE containing only 8 spheres. Even if none of these different modeling approaches are able to describe the whole set of experimental data, they can be considered as an analysis tool in order to understand the parameters that influence the mechanical reinforcement of nanocomposites. However, the input parameters are often delicate to identify and quantify. Moreover they are generally inefficient to describe reinforcement for high filler content and when strong filler-filler interactions create a rigid filler structure in the polymer. For instance, this has been reported for cellulose whiskers and carbon nanotubes filled materials $[50,51]$ and was characterized by a filler content threshold (the percolation threshold) above which the composite properties (mechanical, electrical...) were steeply evolving. While contacts between inclusions are hard to implement in FE model, classical series-parallel model with an additional percolation concept has been proposed by Ouali et al. [52]. This model considers two types of fillers in 
the composite. The first type is the percolating fillers that create continuous paths throughout the composite material, and the second type is the dispersed fillers that do not participate in these paths. These parts of the overall materials are then combined with the polymer matrix following a series-parallel model (Figure 7). The elastic modulus of the nanocomposite is given by equation (2):

$$
\begin{gathered}
E_{\text {composite }}=\frac{\left(1-X_{\infty}^{2}+X_{\infty} X_{\text {filler }}\right) \cdot E_{\text {contact }} E_{\text {polymer }}+X_{\infty}\left(1-X_{\text {filler }}\right) E_{\text {contact }}^{2}}{E_{\text {polymer }}\left(X_{\text {filler }}-X_{\infty}\right)+E_{\text {contact }}\left(1-X_{\text {filler }}\right)} \\
\text { with }\left\{\begin{array}{l}
X_{\text {filler }} \leq X_{\text {percolation }} \Rightarrow X_{\infty}=0 \\
X_{\text {filler }} \geq X_{\text {percolation }} \Rightarrow X_{\infty}=X_{\text {filler }}\left(\frac{X_{\text {filler }}-X_{\text {percolation }}}{1-X_{\text {percolation }}}\right)^{0.4}
\end{array}\right.
\end{gathered}
$$

where $\mathrm{E}_{\text {composite }}$ and $\mathrm{E}_{\text {polymer }}$ are the elastic moduli of the composite and the polymer respectively. $\mathrm{E}_{\text {contact }}$ accounts for the stiffness of the filler-filler interactions. $\mathrm{X}_{\text {filler }}$ is the volume fraction, $\mathrm{X}_{\infty}$ is the volume fraction of fillers that participate in the percolating network and $\mathrm{X}_{\text {percolation }}$ is the percolation threshold. Though its simplicity, such approach has shown its efficiency to predict modulus of composites in which the filler-filler interaction are much stiffer than the polymer matrix $[50,51]$.

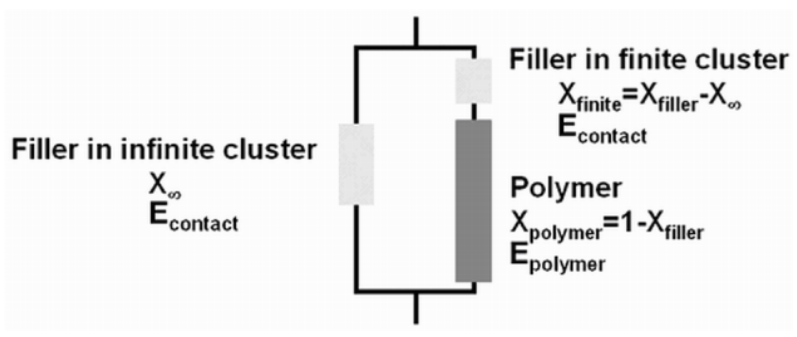

Figure 7: Series/parallel model extended with a percolation concept

To analyze experimental data presented in the previous part, FE modeling has been first performed. Here, two Representative Volume Element (RVE) geometries are considered (Figure 8): cellular (Cell) and randomly dispersed (Dispersed) in order to describe the dispersion of clays in both series. The Cell geometry contains eight full platelets placed on each truncated faces and six half platelets (same diameter but half thickness) placed on each faces of the cubic RVE so that a periodic bound condition is fulfilled and. No contacts are defined between the platelets. The Dispersed geometry is defined as degenerated Cell 
geometry in that the number and orientation of the platelets are kept and the coordinates are changed in order to remove the presence of edge to edge vicinity of platelets. The size ratio of the RVE is comparable to that of the latex particle compared to the Laponite platelet.

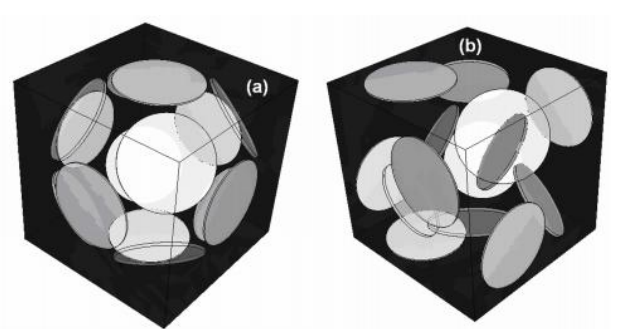

Figure 8: Cell (a) and Dispersed (b) RVE geometries (11 platelets of aspect ratio 30) ;

FE calculations are conducted using the commercial software ABAQUS. The elements used are 4 node 3D linear tetrahedron elements (C3D4) for the matrix and Lapnite platelets are considered as rigid shells. A uniform strain is applied on the face of the elementary cell. The clay volume fraction and the aspect ratio are adjusted by changing the thickness and radius of the platelets while the RVE volume is kept constant. Calculation is made with platelets of aspect ratio of 30 and a matrix of elastic modulus of $0.3 \mathrm{MPa}$. Unfortunately, this configuration is limited to a the volume fraction of $2 \mathrm{vol} \%$ (corresponding to $5 \mathrm{wt} \%$ ). Up to this level, both RVE give the same modulus values. As shown in Figure 9, the modeled curve (black dashed line) fit correctly experimental data (circles and triangles). They also indicate that up to this clay content, there is no need to consider filler-filler contacts.

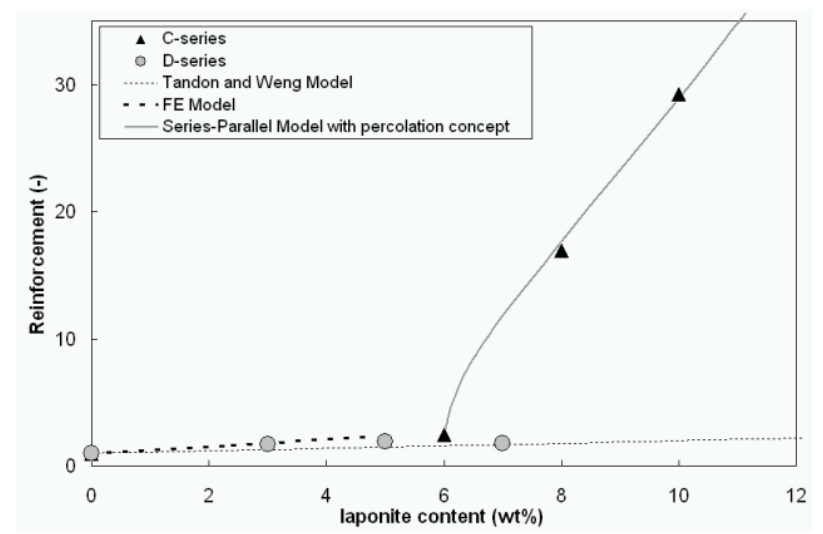

Figure 9: Experimental Reinforcement ( $\left.E_{\text {composite }} / E_{\text {matrix }}\right)$ for both $C$ and $D$ series where $E$ (Elastic modulus) is the initial slope of the strain-stress curve). Comparison with a 
classicalmean field approach (Tandon and Weng [44]) and a series-parallel model with percolation concept and a FE prediction.

The same FE approach has been extended to the prediction of the mechanical behavior of the composites at large deformation. Using the DISPERSED RVE geometry with a polymer matrix described as an elasto-plastic behavior with an elastic modulus of $0.3 \mathrm{MPa}$ and a plastic plateau at $0.08 \mathrm{MPa}$. The results plotted in Figure 10 shows a calculation with platelets of aspect ratio 20 and 30 and a clay content of $2 \mathrm{wt} \%$.

Simulations and experimental data fairly correlate and the elastic modulus (initial slope) is best described for an aspect ratio of 20 . This could be highlighting some clay aggregation during the synthetic process leading to a decrease of aspect ratio (compared to 30 for fully exfoliated clays in theory). In addition, the softening of the curve is sharper in the model than in the experimental data, this probably comes from the pure elasto-plastic behavior chosen for the matrix in the model.

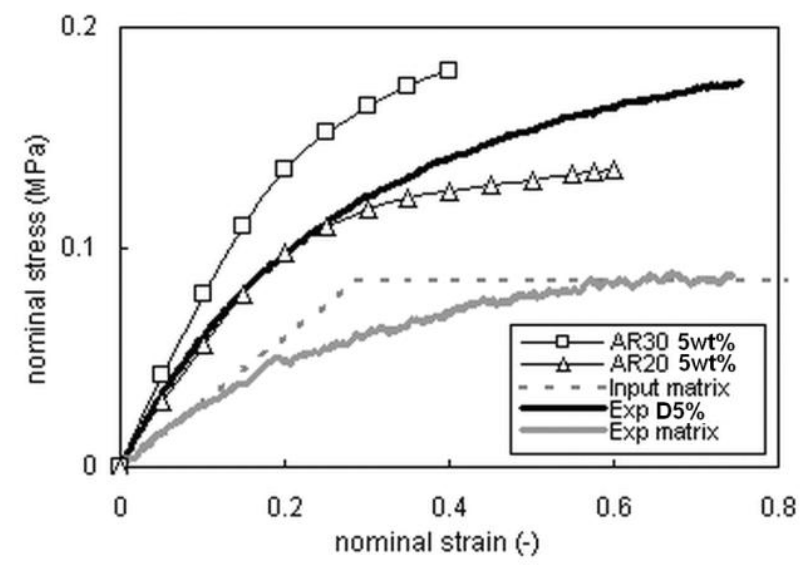

Figure 10: comparison of the mechanical behavior calculated by the FE model and the experimental data (sample D5\%).

While the mechanical behavior of the samples with low clay contents is fairly well described by this FE approach the modeling of the reinforcement of the samples with high clay contents requires introducing the role of clay contacts. Indeed, in Figure 9, the comparison between mean field approach predictions (here, Tandon and Weng model [44] in grey line) and the experimental data (circles and triangles) indicates that the modulus of the $\mathrm{C}$ samples (at clay content above $4 \mathrm{wt} \%$ ) are largely underestimated. Actually, the Laponite percolation threshold depends on the coverage of the latex particles and therefore on the clay exfoliation and also on the size of the latex particles. Indeed, full exfoliation would lead to the maximum amount of Laponite platelets 
available for coverage. In addition, large latex particles lead to smaller latex surface to cover. Therefore good clay exfoliation and large latex particles are two parameters that lower the percolation threshold. Thus, the high reinforcement observed for these Csamples is likely due to a Laponite percolation through interactions between clays from neighboring latex particles after film formation. For D-samples, the clay platelets are preferentially embedded in the polymer particles, therefore the percolation threshold must be high and was not reached due to limitations in the miniemulsion processing route. The Ouali approach and equation 12 have been used to model the modulus evolution of the $\mathrm{C}$-series above the percolation threshold $\left(\mathrm{X}_{\text {percolation }}\right)$ estimated from the DMA results (around 2.5vol\%). For this calculation, the elastic modulus of the polymer is again set at $0.3 \mathrm{MPa}$. $\mathrm{E}_{\text {contact }}$ representing the stiffness of the Laponite-Laponite contacts, was set to 3GPa. Figure 9 shows that this model (black line) fairly correlates the reinforcement of experimental data from $\mathrm{C}$ samples (black triangles) for high Laponite contents.

Thus, both experimental data and modeling approaches evidenced the key role played by the contacts in terms of reinforcement and damage. To enrich this comparison of the impact of morphology on material properties, the water uptake behavior is under the scope of this study. Indeed, from the coatings application point of view, this aspect of the material behavior is of primary importance and thus will be developed hereafter.

\subsection{Water uptake behavior}

The surfactant presence is known to have small effects on the overall mechanical properties i.e. no specific effects on mechanical properties of waterborne films with low amount of surfactant have been reported in literature. However, as far as water uptake is concerned, the role of surfactant cannot be neglected anymore. Actually, the surfactant molecules used to stabilize the latex remain in the final material and present the specificity of being both hydrophobic and hydrophilic. In films obtained from pure polymer latex, Kientz et al [53] counted three primary outcomes for surfactant molecules: dissolution in the polymer, formation of a continuous membrane at the internal polymer/polymer interfaces and formation of islands of surfactant throughout the film and interfaces (film/air and film/substrate). Water absorption of films formed from latex is a critical characteristic for coatings application in particular for exterior use. At the moment, latex paints are mainly used in interior decorative coatings and 
solvent-borne coatings are still preferred for exterior use. In their review on water transport in organic coatings, Van der Wel et al. [54] listed the typical sorption curves that are encountered for organic coatings. The most classical is the Fickian sorption curve whose three characteristic features are an initial linear plot of $\mathrm{M}_{\mathrm{t}}$ as a function of $t^{1 / 2}$, a smooth leveling of the sorption curve to a saturation level $\mathrm{M}_{\infty}$ at higher immersion time and a plot of $\mathrm{M}_{t} / \mathrm{M}_{\infty}$ independent of the thickness of the sample. Agarwal. et al. [55] reported that acrylic based latex film after water absorption lost their transparency and observed freeze-fractured samples by Scanning Electron Microscopy. The samples exhibited micron size holes that suggested clusters of water inside the bulk of the film that were associated to residual surfactant lumps inside the film. DMA experiments on wet films showed a slight plasticization effect, however most of the water remains separated from the bulk polymer as an independent entity. Agarwal et al. proposed a qualitative model by diffusion-driven pathways to clusters of surfactant in the material that act as water reservoirs. While it seems clear that the layer of surfactant has to collapse in order to facilitate the close contact between latex particles and the subsequent polymer chain interdiffusion, it seems also reasonable to consider that surfactant path still remains in the film as well as surfactant islands. Hydration of these surfactant salts by water provides ample driving force for prolonged water absorption, which is limited only by the bulk strength of the polymer. Enlargement of these hydrated domains due to increasing amounts of water causes them to scatter light leading to the loss of transparency of the film. On the other hand, Jubete et al. [56] reported a study concerning the addition of talc in carboxylated styrene butadiene rubber (c-SBR) based waterborne paint. It was observed that adding 20phr of talc ( $10 \mu \mathrm{m}$ diameter) to the c-SBR matrix reduced the water uptake relative to the unfilled matrix. This was attributed to a barrier effect associated with the large platy particle that physically blocked the penetration of water molecules. However, for talc levels greater than 20phr, this barrier effect was counteracted by percolation where contact between talc particles provided easier route for water molecules to diffuse along the interfacial region between filler and matrix. The addition of $\mathrm{TiO}_{2}(240 \mathrm{~nm}$ diameter) to the talc/polymer system was found to decrease the water absorption of the composite, probably due to an increase of tortuosity that hinders water diffusion.

In the systems coming from the E.U. project, several types of "salts" are used during latex polymerization with an average amount around $2 \mathrm{wt} \%$ (salts content for each 
recipe are given in Table 2). Actually, as suggested by literature, in addition to the surfactant content and location throughout the film, contacts between clay platelets are likely to be crucial. For example, one can imagine specific interactions between the surfactant molecules and the clay surfaces or confinement in the clay inter-galleries. The presence of Laponite platelets on the surface of the latex particles in the $\mathrm{C}$ series could hinder the mobility of the surfactant and therefore limit the formation of surfactant islands. On the other hand, the surfactant molecules in the D-samples should behave like in a blank sample considering that the Laponite clays are embedded and therefore the main interactions are surfactant-polymer interactions.

Table 2: List of "salts" and their content in each sample,

\begin{tabular}{llllll}
\hline Sample & $\begin{array}{l}\text { Laponite- } \\
\text { Laponite contacts }\end{array}$ & $\begin{array}{l}\text { Dowfax content } \\
(\mathrm{wt} \%)\end{array}$ & $\begin{array}{l}\text { DDAB } \\
(\mathrm{wt} \%)\end{array}$ & $\begin{array}{l}\text { Peptizer } \\
(\mathrm{wt} \%)\end{array}$ & $\begin{array}{l}\text { Salt content } \\
(\mathrm{wt} \%)\end{array}$ \\
\hline C 0\% & - & 1.9 & - & 0.0 & 1.9 \\
C 6\% & Yes & 1.7 & - & 0.4 & 2.1 \\
C 8\% & Yes & 1.7 & - & 0.5 & 2.2 \\
C 10\% & Yes & 1.6 & - & 0.5 & 2.1 \\
D 0\% & - & 1.8 & 0.0 & - & 1.8 \\
D 3\% & No & 1.8 & 0.2 & - & 2.0 \\
D 5\% & No & 1.8 & 0.4 & - & 2.2 \\
D 7\% & No & 1.7 & 0.5 & - & 2.2 \\
\hline
\end{tabular}

Water absorption was performed with samples cut out of each polymer film. These sample series are referred as 'fresh' hereafter. Another set of samples were weighed and then left immersed in water for three months. These samples were then dried and reweighed, a difference of $2-3 \mathrm{wt} \%$ was found, which was probably due to a loss of salts initially present in the films. Water sorption experiments were performed on those samples referred as 'washed' hereafter. Water uptake experiments have been performed on both fresh and washed specimens for both $\mathrm{C}$ and D series. The relative mass uptake was determined using equation (3):

$$
M_{t}(\%)=\left(\frac{W_{t}-W_{0}}{W_{0}}\right) \cdot 100
$$

Where $\mathrm{W}_{\mathrm{t}}$ and $\mathrm{W}_{0}$ are respectively the instantaneous and initial weights 
The sorption curves (relative mass uptake vs square root of immersion time (up to ca. 500hours)) are plotted in Figure 11a-b for fresh (black signs) and washed (grey signs) specimens for D and C samples respectively. First comment is that once washed, all samples reached less than $20 \mathrm{wt} \%$ mass uptake after ca. $500 \mathrm{~h}$ immersion in all case whereas some of the fresh samples reached up to 50wt.\% mass uptake after the same immersion time. This drop between fresh and washed samples is observed whatever the Laponite nanostructure and whether the film contained clays or not. It is likely due to the fact that the washing step induced the dissolution of salts from the polymer film to the water bath. The removal of surfactant after the prolonged immersion in water has been confirmed by measurement of the Laponite layer spacing through XRD experiments for both fresh and washed samples. For instance, for the C6\% sample, it was found that the peak at $2 \theta=3^{\circ}$ corresponding to a swollen d-spacing of $2.8 \mathrm{~nm}$ vanished and slightly shifted to the higher angles for the washed sample. Similar results were obtained with the D5\% sample. In addition, the drop between fresh and washed samples highlights the fact that, despite their low amount in the film, salts causes a large water uptake compared to the polymer matrix.
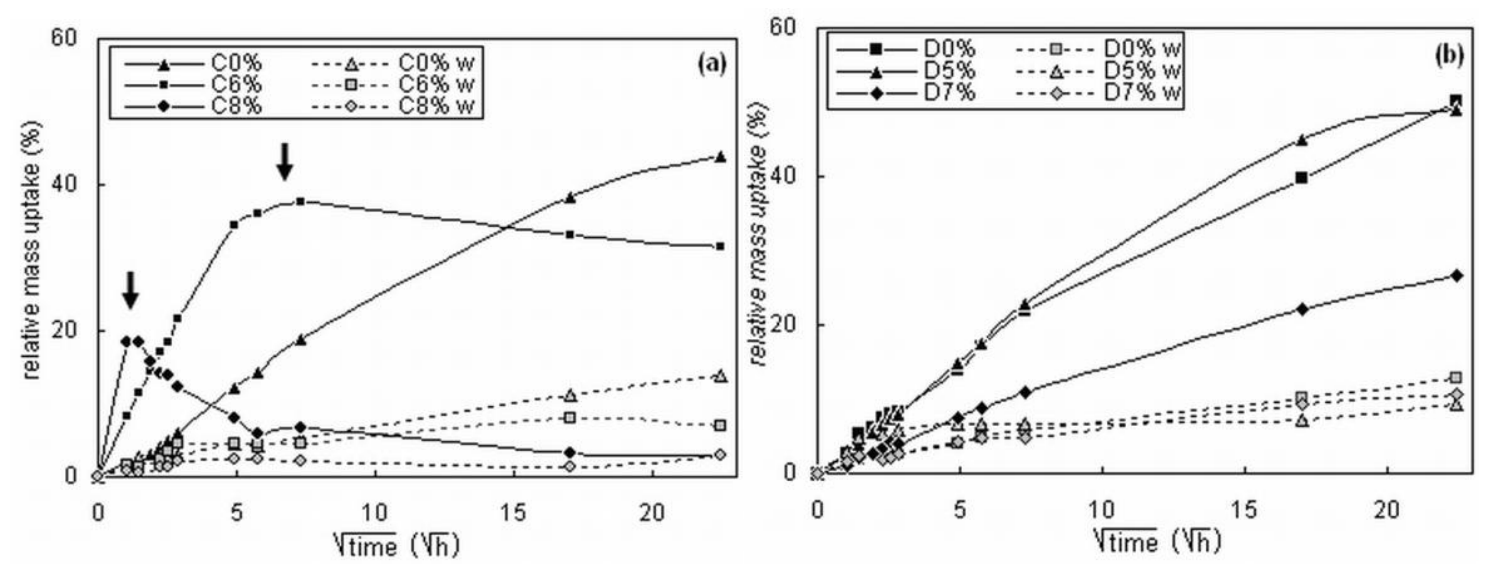

Figure 11: relative water uptake of (a) D-series and (b) C-series for fresh (-) and washed (w) samples. At least two specimens of $15 \mathrm{~mm} \times 10 \mathrm{~mm} \times 0.35-0.45 \mathrm{~mm}$, dried in vacuum at $60^{\circ} \mathrm{C}$ for 24 hours. Immersion in distilled water at room temperature. Specimens periodically removed from water, dried with filter paper and immediately weighed with a precision of $0.01 \mathrm{mg}$ before returning to the water bath.

Besides, peculiar features are visible on the sorption curves for $\mathrm{C}$ samples fresh compared to the D ones. Indeed, C8\% plot exhibits a maximum peak at low immersion time meaning that a fast dissolution of salts from the film to the water bath is occurring. In the washed specimens, such peak is not visible as the salts have been already 
removed prior to the experiment. The absence of such feature for the D series means that exudation of salts from D samples is likely occurring slowly in time compared to the $\mathrm{C} 8 \%$ sample. Moreover, the initial slope of the $\mathrm{C} 8 \%$ fresh specimens that contain clays is much higher than the fresh blank specimen. Conversely, for the D samples, the initial slope of the sorption curve is of the same order for both washed and fresh specimens whether they contain clays or not. Note that the C6\% sample shows an intermediate behavior in between that of the $\mathrm{C} 8 \%$ and that of the $\mathrm{D}$-series, since in this composite, the clay content is close to the percolation threshold. Thus, $\mathrm{C}$ nanostructure increases the kinetics of water absorption due to the presence of Laponite paths through the film that favors the access of water to the salts inside the film. These same Laponite paths are also preferential ways out for the salts to exit from the material as dissolved salts. Thus, these experiments clearly demonstrated that the nanostructure of these films has an impact on the water uptake and surfactant dissolution kinetics and led to the following proposed scheme (Figure 12) concerning both types of nanostructured films before and after being washed.

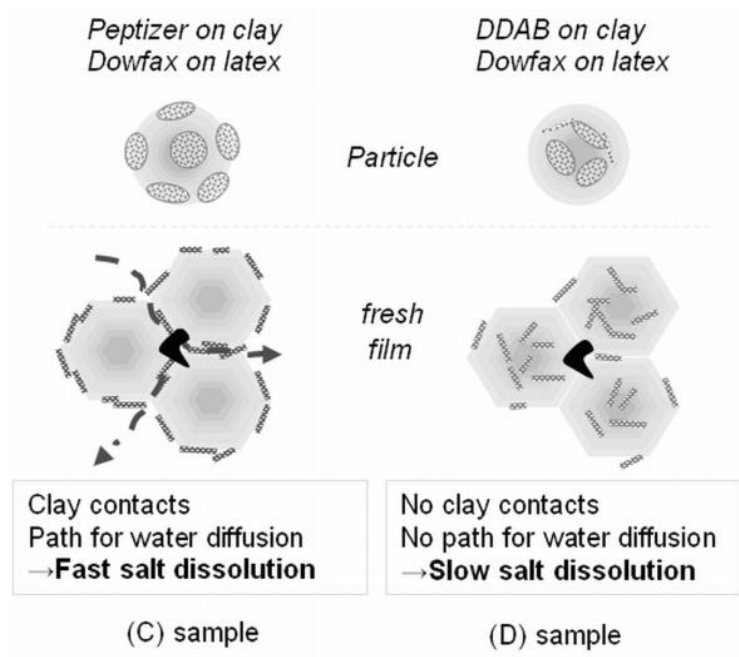

Figure 12: scheme of both types of nanostructured films before and after being washed. The black lump represents a surfactant lump.

At last, it can be noted that even when washed, the Laponite nanostructure has an effect on the water uptake. It seems that the cellular nanostructure tends to hinder the water absorption. The mass uptake after 300hours for washed samples versus the Laponite content is plotted in Figure 13. The trend for the D samples is a slight decrease for increasing Laponite content. For the $\mathrm{C}$ samples, the decrease is larger, i.e. at comparable Laponite contents, i.e. the $\mathrm{C}$ samples exhibit a lower water absorption. Two different 
explanations can be proposed. First, once washed, the polymer is the higher absorber in the system and the unswollen clays form obstacles to the water swelling due to intrinsic barrier properties of "washed clays". The barrier effect hindering the diffusion of water from a cell to another can also be due to some mechanical effect: the higher modulus associated with the cellular nanostructure limits the deformation ability of the polymer matrix.

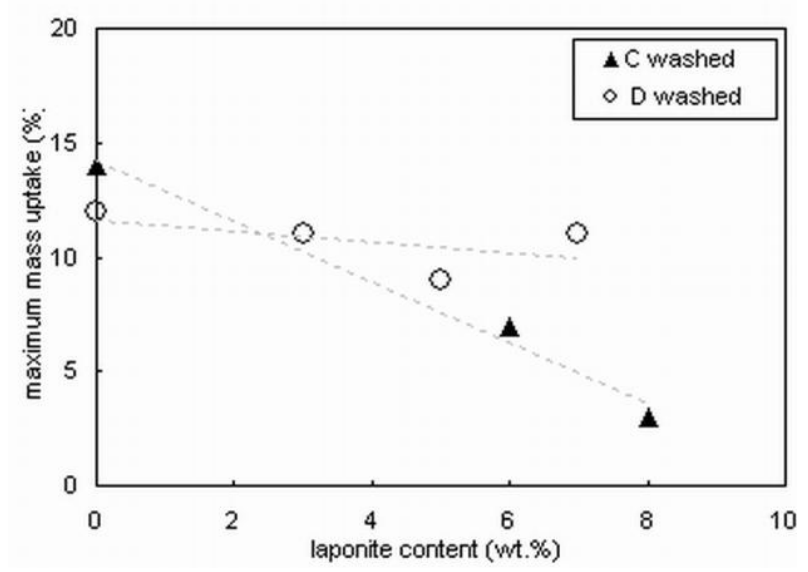

Figure 13: maximum water uptake of washed samples of both nanostructures.

\section{Conclusion}

Motivated by environmental and health aspects, waterborne latexes has been widely explored for decades. In the literature concerning nanocomposites synthesized by in-situ polymerization, an increasing number of works is about heterophase polymerization. Compared to bulk polymerization and solution polymerization, this processing route ensures a good dispersion of the clays in the polymer matrix after evaporation of water. Moreover, there is no problem of increased viscosity at the end of polymerization like in bulk polymerization and this technique do not suffer of drawbacks of dilution (lower kinetics) like solution polymerization. For now, a lot of works have focused on the clay dispersion quality and take advantage of the advances in clay surface treatments. However, it appears that emulsion polymerization is not adequate for clay encapsulation: the obtained nanostructure in that case consists of clays covering the surface of latex particles. On the contrary, miniemulsion polymerization enables to create two different types of nanostructures: First, the clays covering the surface of the latex particles as previously and second the clays embedded inside the latex particle. This ability to produce nanocomposites with similar clay dispersion but radically different nanostructures is one of the breakthrough advances of nanocomposite 
production by miniemulsion polymerization. This study aimed at comparing new nanocomposite materials processed through this latex technology. Two new synthetic routes were developed to obtain a good dispersion of Laponite throughout the nanocomposite film even if a complete exfoliation of the clay was not reached. In Route I (C-samples), the clay fillers were located on the surface while in Route II (D-samples), the clay platelets were encapsulated inside the polymer particles. The film formed from those latexes presented very different nanostructures. The first series of nanocomposite materials presented a network of Laponite able to percolate at higher clay contents, whereas interactions between clays were limited in the D-samples due to their confinement inside the individual polymer particles. Large effects were observed on the mechanical properties. Indeed, due to the presence of interaction between clay particles in the C-samples, high reinforcement factors were observed from ca. 3vol\% Laponite content whereas lower reinforcement was observed for the D-samples. Different modeling approaches enable to analyze the reinforcement effects. Moreover, due to the localization of stress at the Laponite edges in the C-samples, mechanical damage occurred whereas the D-samples exhibited a viscoelastic behavior similar to a blank polymer matrix. The nanostructure leads also to strong difference in the water uptake. The salts are the main cause for water uptake for fresh samples. The removal of these salts is faster when the nanostructuration leads to clay paths throughout the films. Thus, the main drawback of latex technology for its further application in coatings is the presence of surfactant molecules in the system. However, under adequate conditions, surfactant-free miniemulsion polymerization could be performed, taking advantage of the stabilization provided by the adsorption of clays on the surface (Pickering emulsion). The optimisation of this new route is the following step to produce new materials for industrial application that combine a sustainable process and improved properties.

\section{Acknowledgements}

This work was funded by the EC Framework 6 Integrated Project, NAPOLEON, under contract No. IP 011844-2. 


\section{References}

1. Chazeau L, Gauthier C, Vigier G, and Cavaillé JY. Nanocomposites. In: Nalwa HS, editor. Handbook of Organic-Inorganic Hybrid Materials and Nanocomposites, vol. 2. Japan, 2003.

2. Kojima Y, Usuki A, Kawasumi M, Okada A, Fukushima Y, Kurauchi T, and Kamigaito O. Journal of Materials Research 1993;8(5):1185-1189.

3. Alexandre M and Dubois P. Materials Science and Engineering R: Reports 2000;28(1):1-63.

4. Schmidt D, Shah D, and Giannelis EP. Current Opinion in Solid state and Materials Science 2002;6:205-212.

5. Ray SS and Okamoto M. Progress in polymer science 2003;28(2003):1539-1641.

6. Tseng CR, Wu JY, Lee HY, and Chang FC. Polymer 2001;42(25):10063-10070.

7. Sur GS, Sun HL, Lee, T.J., Lyu SG, and Mark JE. Colloid and Polymer Science 2003;281(11):1040-1045.

8. Ma JZ, Hu J, and Zhang ZJ. European Polymer Journal 2007;43(10):4169-4177.

9. Han B, Ji G, Wu S, and Shen J. European Polymer Journal 2003;39(8):1641-1646.

10. Steward PA, Hearn J, and Wilkinson MC. Advances in Colloid and Interface Science 2000;86(3):195-267.

11. Keddie JL. Materials Science \& Engineering R-Reports 1997;21(3):101-170.

12. Chevalier Y, Pichot C, Graillat C, Joanicot M, Wong K, Maquet J, Lindner P, and Cabane B. Colloid and Polymer Science 1992;270(8):806-821.

13. Visschers M, Laven J, and German AL. Progress in Organic Coatings 1997;30(1-2):39-49.

14. Jensen DP and Morgan LW. Journal of Applied Polymer Science 1991;42(10):2845-2849.

15. Al-Mukhtar M, Touray JC, and Bergaya F. Comptes Rendus De L Academie Des Sciences Serie Ii Fascicule a-Sciences De La Terre Et Des Planetes 1999;329(4):239-242.

16. Utracki LA, Sepehr M, and Boccaleri E. Polymer Advance Technology 2007;18:1-37.

17. Lee DC and Jang LW. Journal of Applied Polymer Science 1996;61(7):1117-1122.

18. Noh MH and Lee DC. Journal of Applied Polymer Science 1999;74(12):2811-2819.

19. Yang J, Fan H, Bu Z, and Li B. Acta Polymerica Sinica 2006;6.

20. Ogawa M, Okutomo S, and Kuroda K. Journal of the American Chemical Society 1998;120(29):7361-7362.

21. Negrete-Herrera N, Persoz S, Putaux JL, David L, and Bourgeat-Lami E. Journal of nanoscience and Nanotechnology 2006;6:421-431.

22. Negrete-Herrera N, Putaux JL, and Bourgeat-Lami E. Progress in Solid State Chemistry 2006;34(2-4):121-137.

23. Rubio J and Kitchener JA. Journal of Colloid and Interface Science 1976;57(1):132-142.

24. Ogata N, Kawakage S, and Ogihara T. Journal of Applied Polymer Science 1997;66(3):573-581.

25. Shen YH. Chemosphere 2000;41(5):711-716.

26. Schork FJ, Luo YW, Smulders W, Russum JP, Butte A, and Fontenot K. Miniemulsion polymerization. Polymer Particles, vol. 175, 2005. pp. 129-255.

27. Manea M, Chemtob A, Paulis M, de la Cal JC, Barandiaran MJ, and Asua JM. Aiche Journal 2008;54(1):289-297.

28. Cauvin S, Colver PJ, and Bon SAF. Macromolecules 2005;38(19):7887-7889.

29. Tong Z and Deng Y. Industrial and Engineering Chemistry Research 2006;45(8):2641-2645.

30. Moraes RP, Santos AM, Olivelra PC, Souza FCT, Do Amoral M, Volera TS, and Demarquette NR. Macromolecular Symposia 2006;245:106-115.

31. Sun QH, Deng YL, and Wang ZL. Macromolecular Materials and Engineering 2004;289(3):288295.

32. Samakande A, Sanderson RD, and Hartmann PC. Journal of Polymer Science, Part A: Polymer Chemistry 2008;46(21):7114-7126.

33. Brahimi B, Labbe P, and Reverdy G. Langmuir 1992;8(8):1908-1918.

34. Guinier A and Fournet G. Smal l-angle scattering of X-rays. New York, 1955.

35. Kroon M, Vos WL, and Wegdam GH. International Journal of Thermophysics 1998;19(3):887894.

36. Masenelli-Varlot K, Vicier G, Vermogen A, Gauthier C, and Cavaillé JY. Journal of Polymer Science, Part B: Polymer Physics 2007;45(11):1243-1251.

37. Vermogen A, Masenelli-Varlot K, Vigier G, Sixou B, Thollet G, and Duchet-Rumeau J. Journal of Nanoscience and Nanotechnology 2007;7(9):3160-3171.

38. Faucheu J, Gauthier C, Chazeau L, Cavaillé JY, Mellon V, and Bourgeat-Lami E. Journal of Colloid and Interface Science submitted.

39. G'sell C, Hiver JM, Dahoun A, and Souahi A. Journal of Materials Science 1992;27:5031-5039. 
40. Lo KH, Christensen RM, and Wu EM. Journal of Applied Mechanics-Transactions of the Asme 1977;44(4):663-668.

41. Lo KH, Christensen RM, and Wu EM. International Journal of Solids and Structures 1978;14(8):655-662.

42. Halpin JC and Kardos JL. Polymer Engineering and Science 1976;16(5):344-352.

43. Halpin JC. Journal of Composite Materials 1969;3:732-734.

44. Tandon GP and Weng GJ. Polymer Composites 1984;5(4):327-333.

45. Hine PJ, Lusti HR, and Gusev AA. Composites Science and Technology 2002;62(10-11):14451453.

46. Ahmed J and Jones FR. Journal of Materials Science 1990;25(12):4933-4942.

47. Ji XL, Jing JK, Jiang, W., and Jiang BZ. Polymer Engineering and Science 2002;42(5):983-993.

48. Gusev AA. Macromolecules 2001;34(9):3081-3093.

49. Gusev AA. Journal of the Mechanics and Physics of Solids 1997;45(9):1449-1459.

50. Favier V, Canova GR, Shrivastava SC, and Cavaillé JY. Polymer Engineering and Science 1997;37(10):1732-1739.

51. Dalmas F, Cavaillé J-Y, Gauthier C, Chazeau L, and Dendievel R. Composites Science and Technology 2007;67(5):829-839.

52. Ouali N, Cavaillé JY, and Perez J. Plastics, Rubber and Composites Processing and Applications 1991;16(1):50-60.

53. Kientz E and Holl Y. Colloids and Surfaces a-Physicochemical and Engineering Aspects 1993;78:255-270.

54. Van der Wel GK and Adan OCG. Progress in Organic Coatings 1999;37(1):1-14.

55. Agarwal N and Farris RJ. Journal of Applied Polymer Science 1999;72(11):1407-1419.

56. Jubete E, Liauw CM, and Allen NS. Progress in Organic Coatings 2007;59(2):126-133.

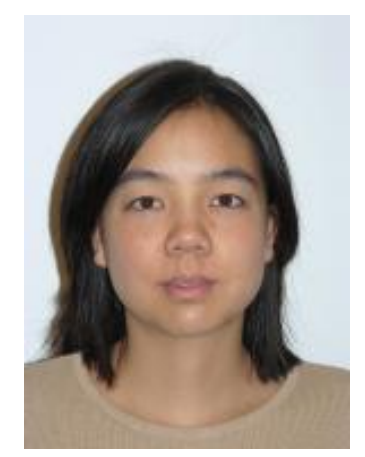

Jenny Faucheu received her Master's degree in 2002 from the European School of Chemistry, Polymers and Materials in Strasbourg. Afterward, she joined the National Institute of Standard and Technology for two years as a guest researcher in the Polymeric Materials group where she studied the ageing of fluorinated polymeric coatings. In 2005, she prepared her PhD. Degree in materials science at the National Institute of Applied Science in Lyon and graduated in 2008. Her doctoral research focused on the relationships between microstructure and mechanical behavior in composite materials. In 2009, she was appointed as lecturer in the Ecole des Mines de Saint-Etienne (EMSE), one of the top 10 French engineering schools. She is involved in exploring the sensory properties of materials. In particular, her research interests include 
the optical, olfactory and haptic properties of materials related to their physical characteristics.

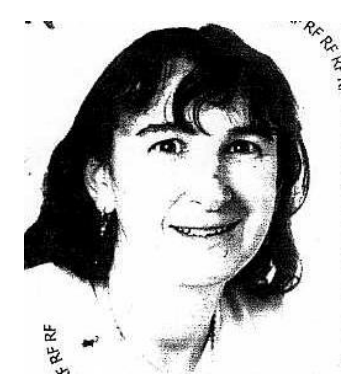

Catherine Gauthier is a research scientist in the MATEIS laboratory of the National Institute of Applied Science in Lyon. She was born in 1965. In 1992, she got her Ph-D degree in materials science at the University Claude Bernard - Lyon I, for her research on the properties-microstructure relationships of unidirectional glass/polyethylene terephtalate composites. She then joined the MATEIS laboratory of the National Institute of Applied Science in Lyon as assistant professor. She obtained a Professor position in the same institute in 2004. Since 2005, she is the head of the Polymer group of MATEIS laboratory. Her current research activity is devoted to mechanical properties-microstructure relationships in polymer based materials, with a special interest for nanocomposites and filled rubbers.

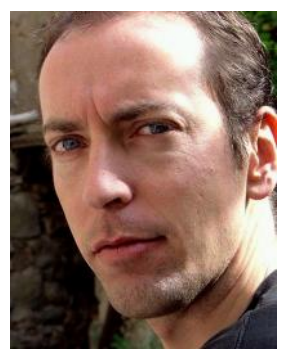

Laurent Chazeau is a research scientist (from CNRS) in the MATEIS laboratory of the National Institute of Applied Science in Lyon. He was born in 1971. In 1998, he got his $\mathrm{Ph}-\mathrm{D}$ degree in materials science at the National Polytechnic Institute of Grenoble, for his research on cellulose whisker/PVC nanocomposites. He then joined the Renssealer Polytechnic Institute, to work as a postdoc with Prof. S.S. Sternstein, on the non linear viscoelasticity of filled elastomer. He then obtained in 1999 his actual position in the polymer group of MATEIS. His current research activity is devoted to mechanical 
properties-microstructure relationships in nanoheterogenous polymer based materials, especially filled elastomers.

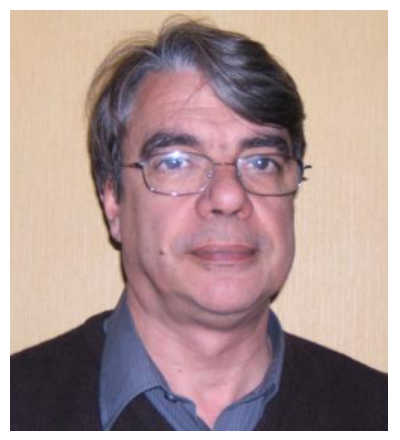

Jean-Yves Cavaillé is currently professor at INSA-Lyon (University of Lyon, France). He got his Engineer Doctor degree in Marseille, working on Field Emission Microscopy. Changing his research domain, he defended his $\mathrm{PhD}$ in 1987, focused on the molecular mobility in amorphous polymers. He became professor in 1989 at the Joseph Fourier University (Grenoble), and constituted a research group on polysaccharide polymers and nanocomposites. In 2000, he took the head of the now socalled Material Science and Engineering Lab (MATEIS). More recently he became codirector of the Science and Engineering Lyon Tohoku international joint lab (ELyT lab). His main field of interest are the physical (and more precisely mechanical and electrical) properties of polymers and amorphous materials in their solid state, and the study of semicrystalline polymers, blends, composites and nanocomposites

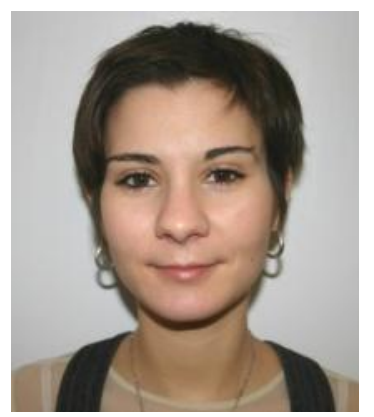

Mellon Véronique was born in 1980 in Tassin la demi lune, France. She obtained her Master degree at ESCPE lyon and a Master degree in Polymer and composite materials at the Claude Bernard University in Lyon. She studied the elaboration and characterization of polymer/Laponite nanocomposite latexes through miniemulsion polymerization and received a Ph.D. degree in materials science from the Claude Bernard University in Lyon in 2009 under the supervision of Dr. Elodie Bourgeat-Lami and Prof. Timothy F.L. MC Kenna. 


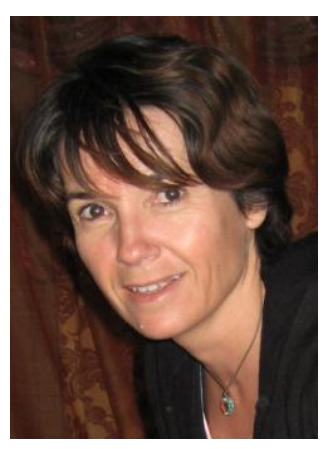

Elodie Bourgeat-Lami was born in 1964 in France. She received a doctor degree from the University of Montpellier II (France) in 1991 under the supervision of Doctor F. Fajula. She is currently Research Director at CNRS in the Laboratory of Chemistry, Catalysis, Polymers and Processes $(\mathrm{C} 2 \mathrm{P} 2)$ headed by Pr. B. Charleux and as on the grafting of polymers to mineral surfaces using the grafting-to and grafting-from techniques. 
Figure(s

Click here to download high resolution image

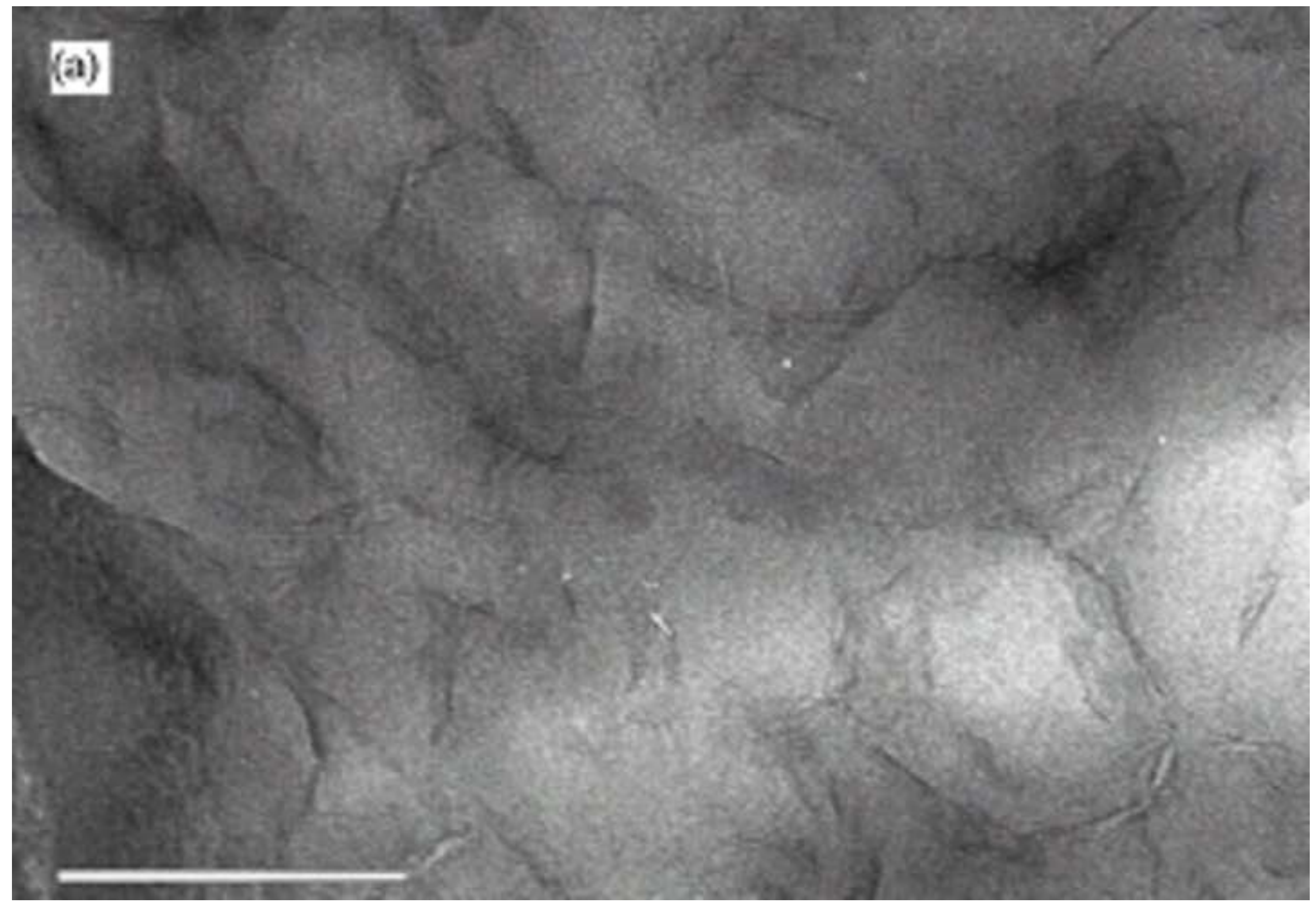


Figure(s

Click here to download high resolution image

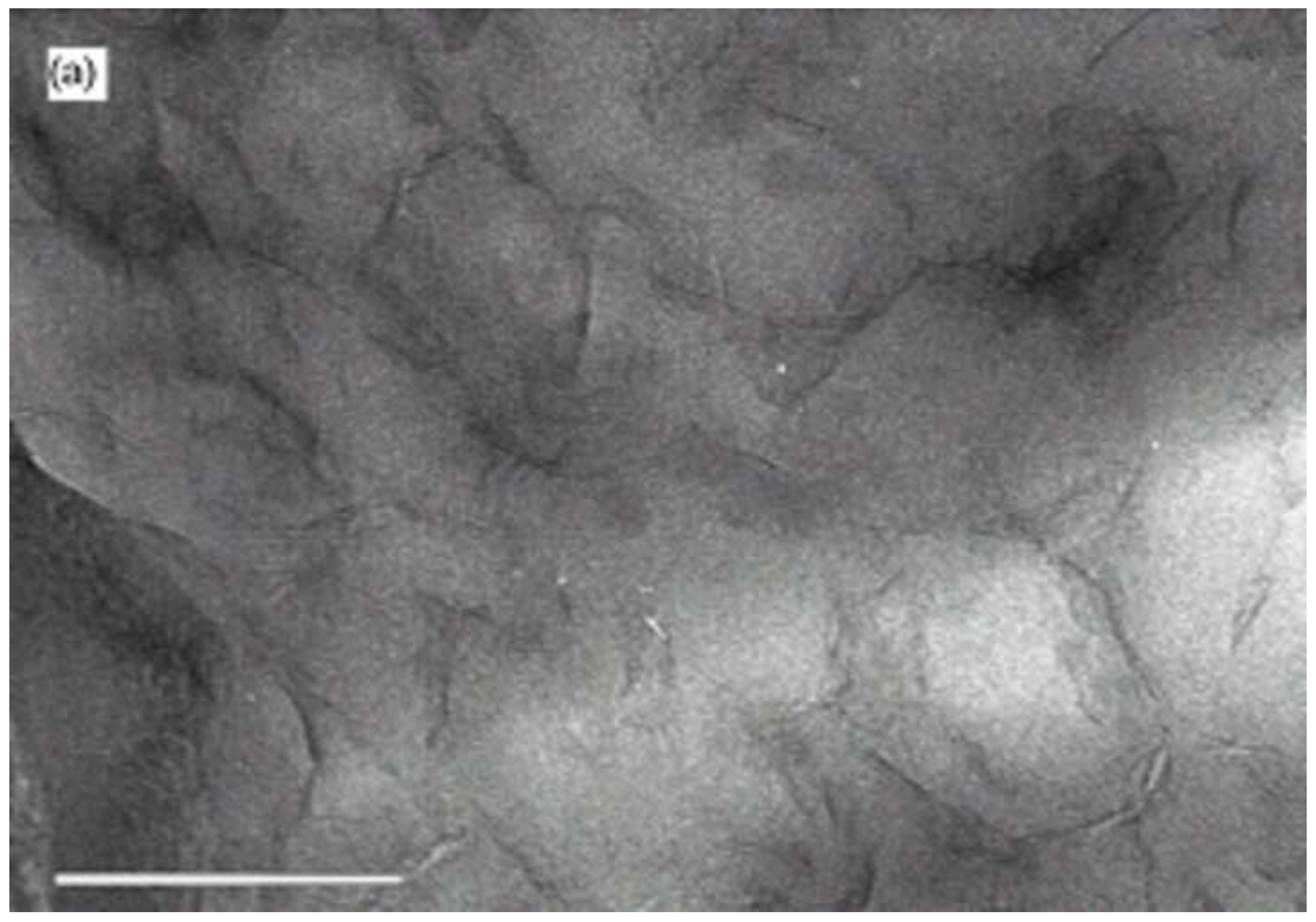

che here to download high resolution inage 


\section{Low clay content}

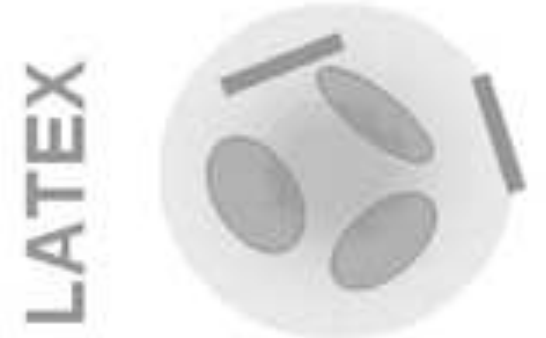

D-series
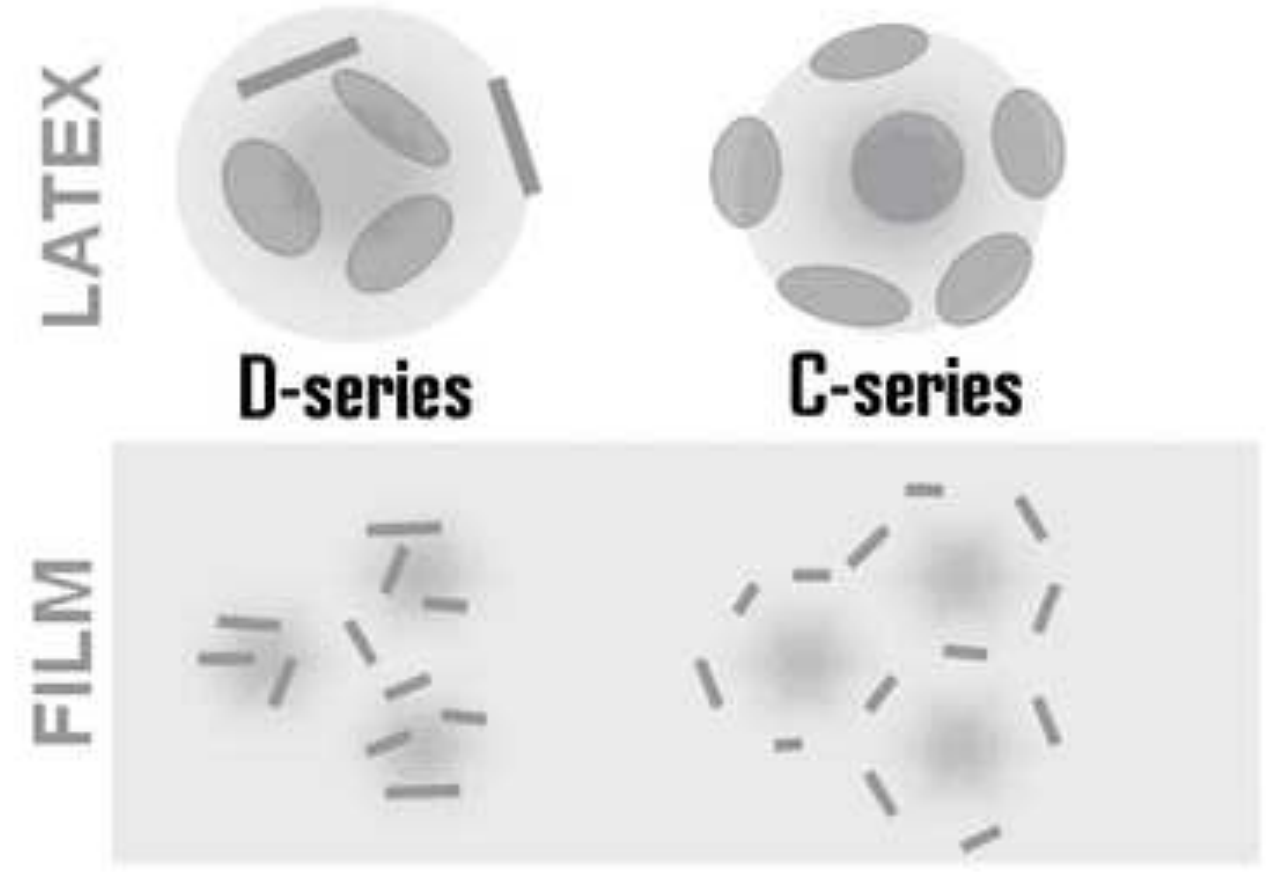

[-series

\section{High clay content}

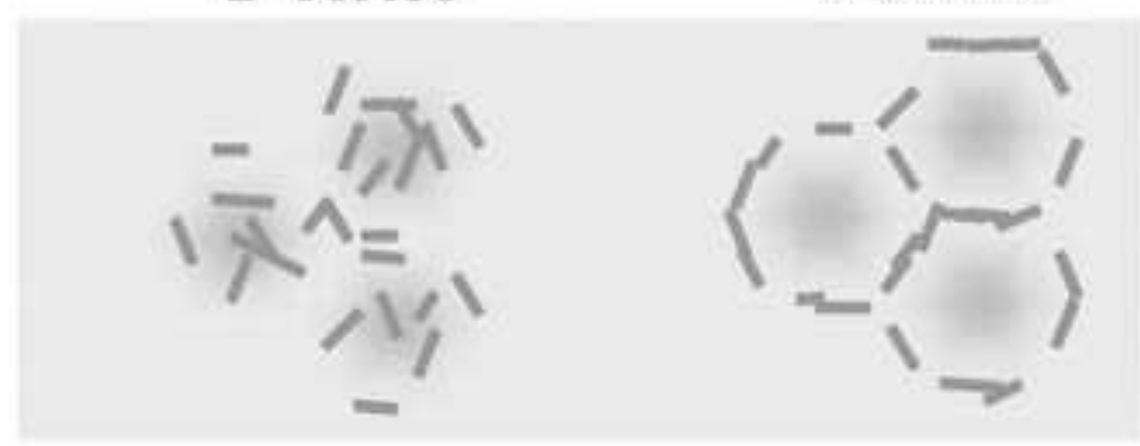

D-series

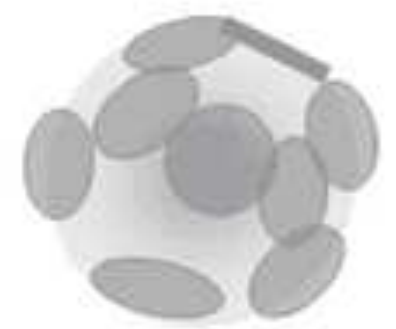

C-series

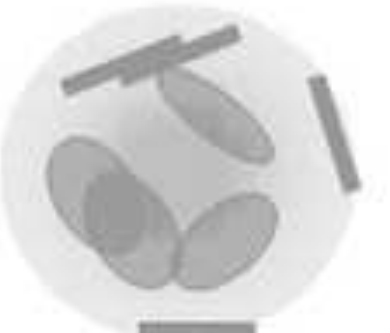




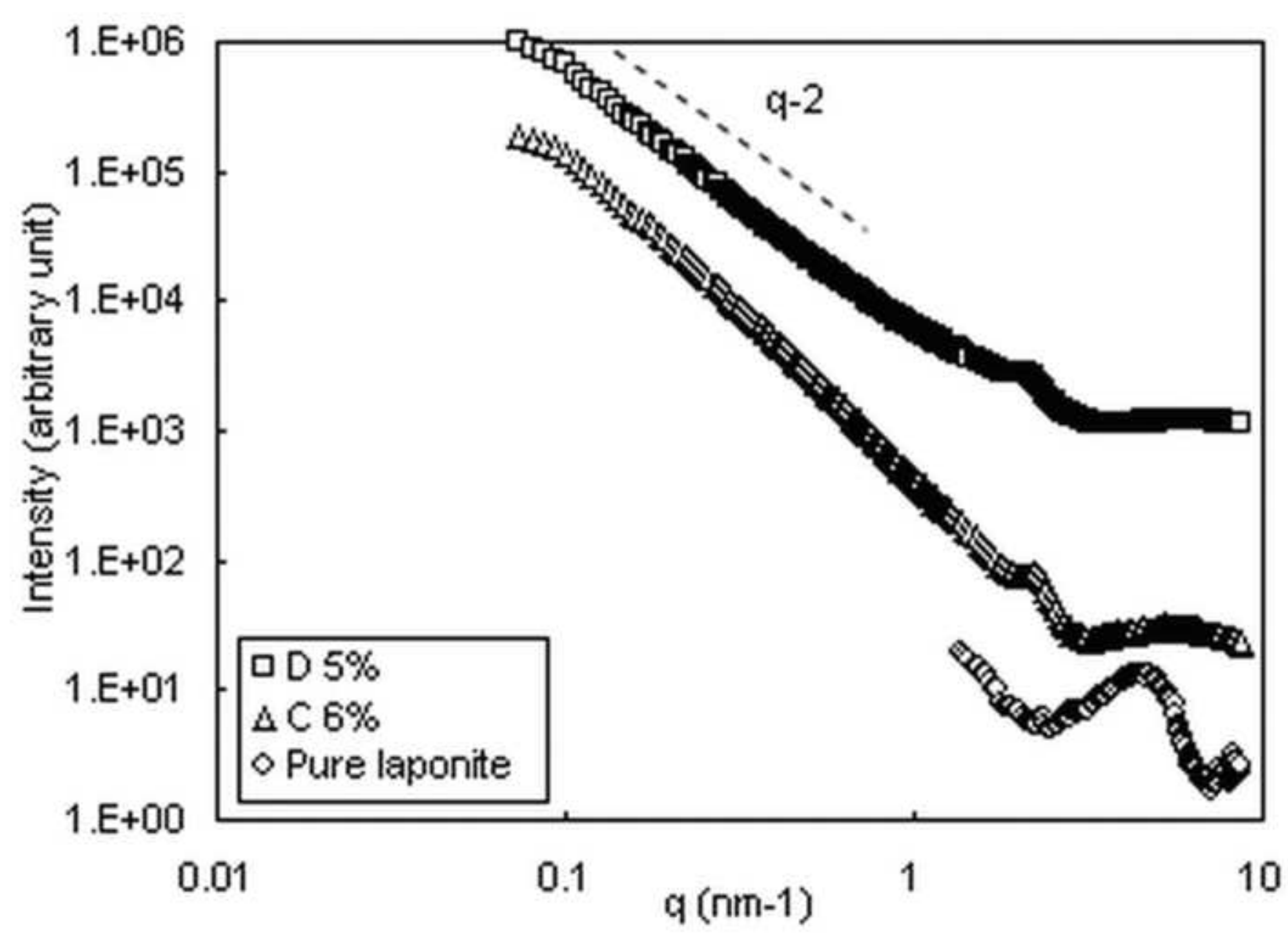




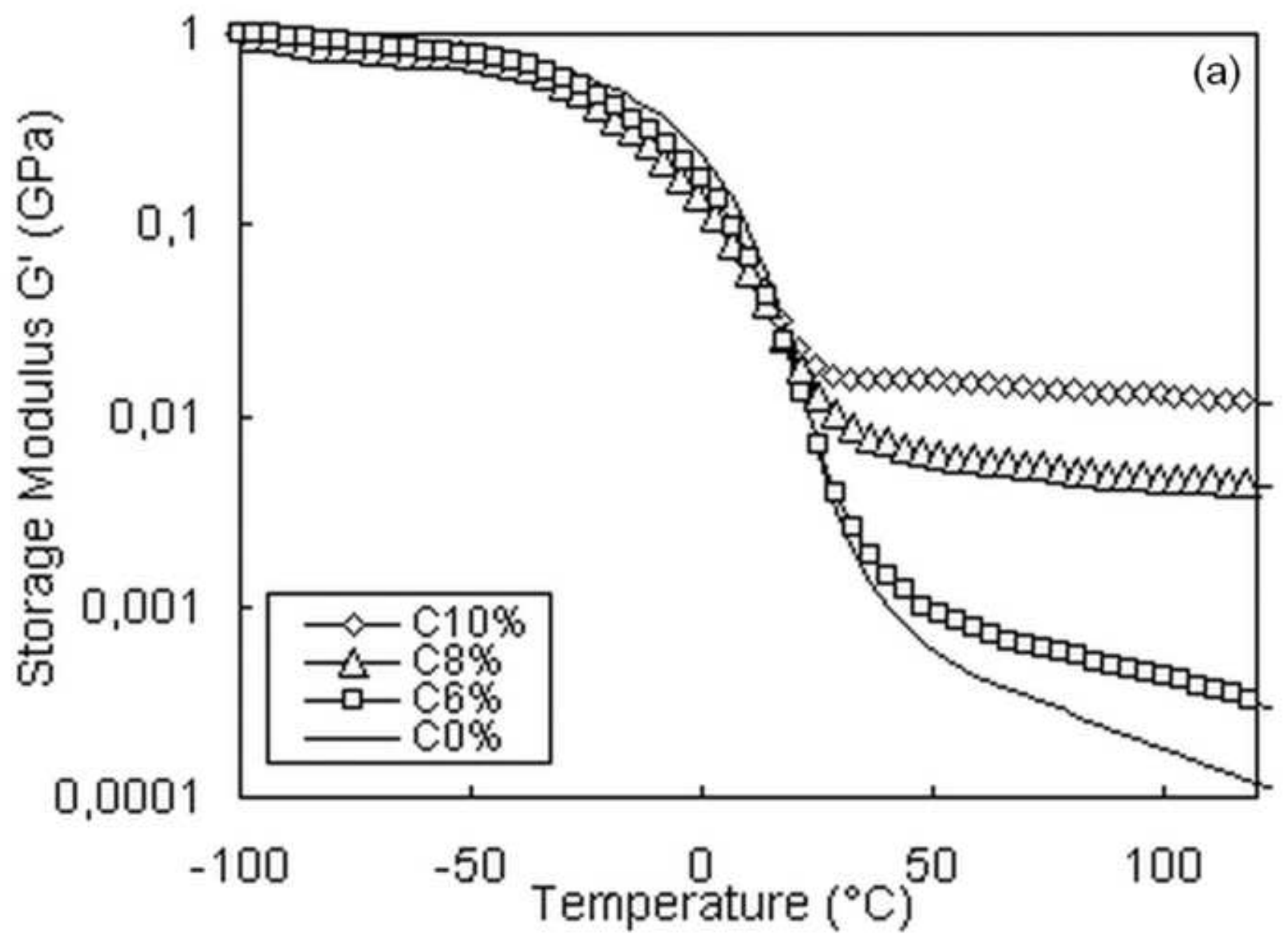




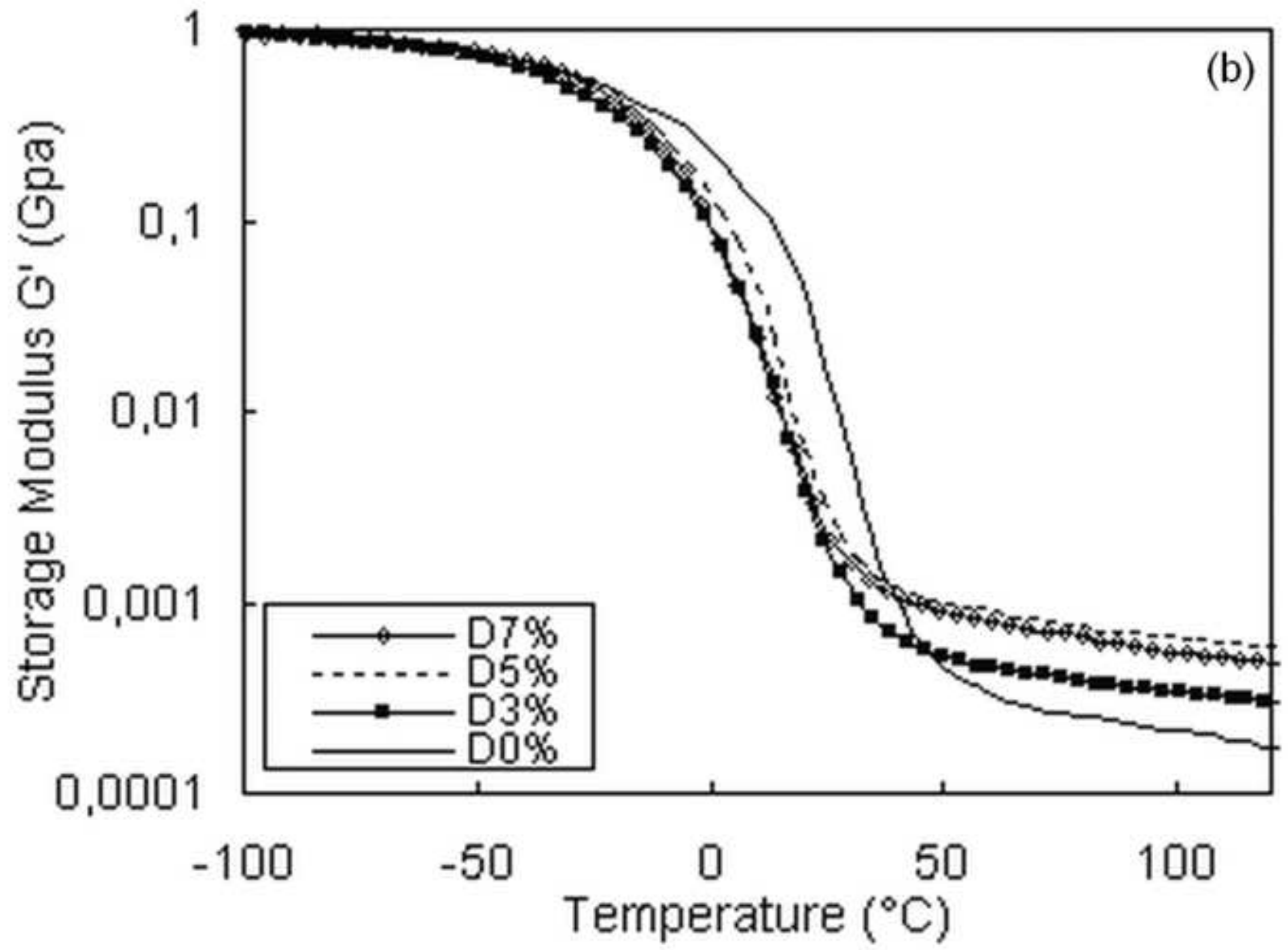


Figure(s)
Click here to download high resolution image

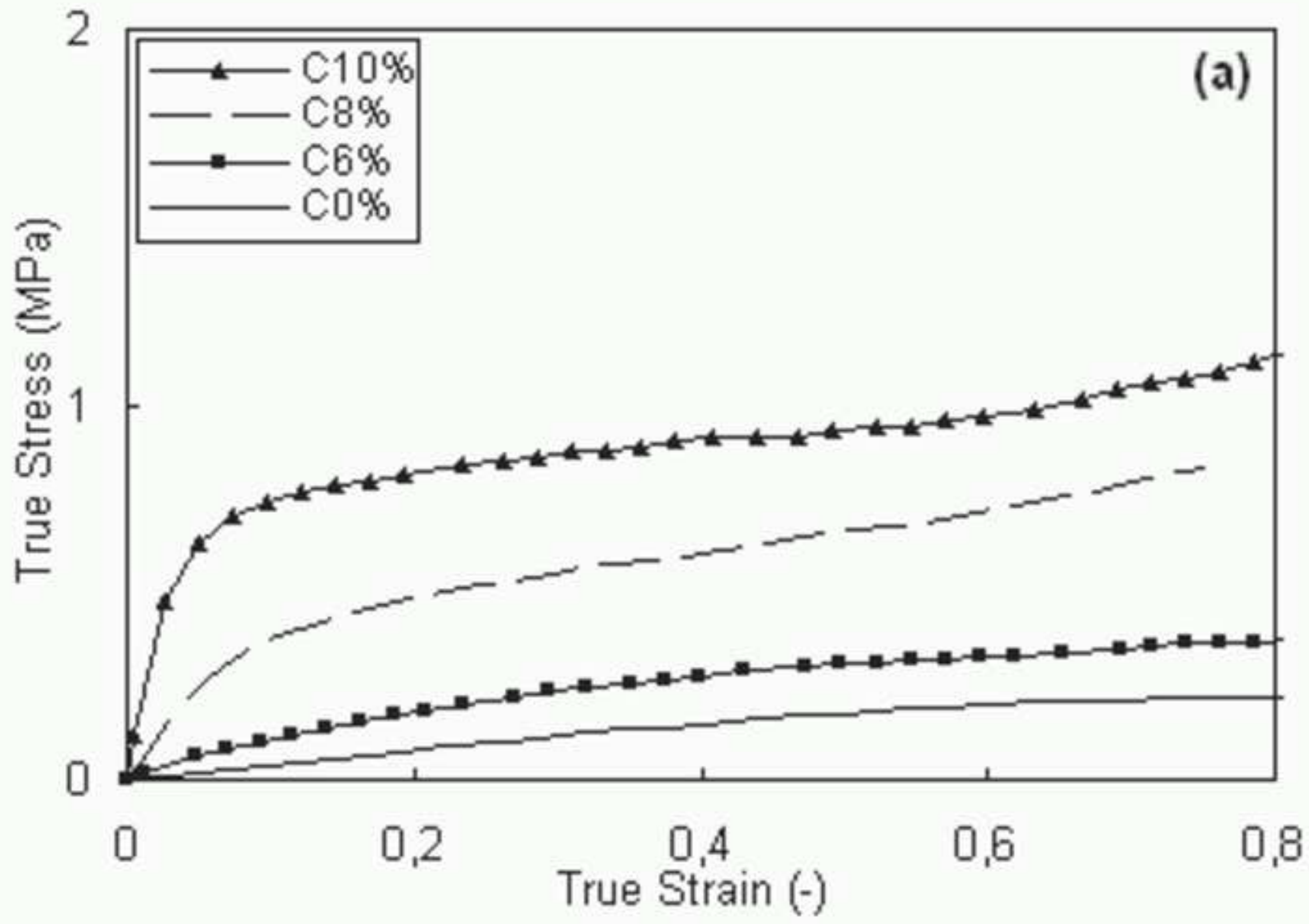




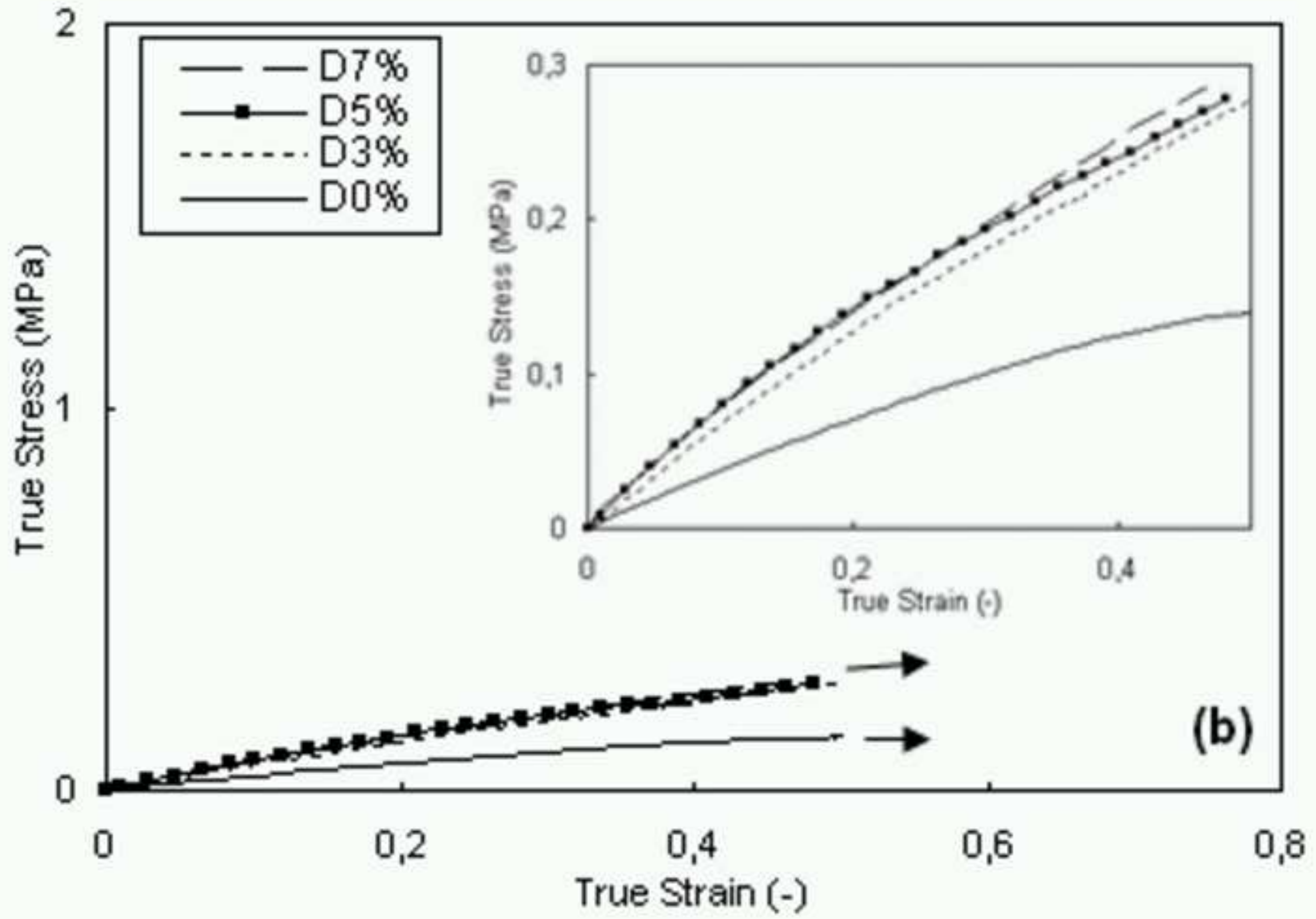



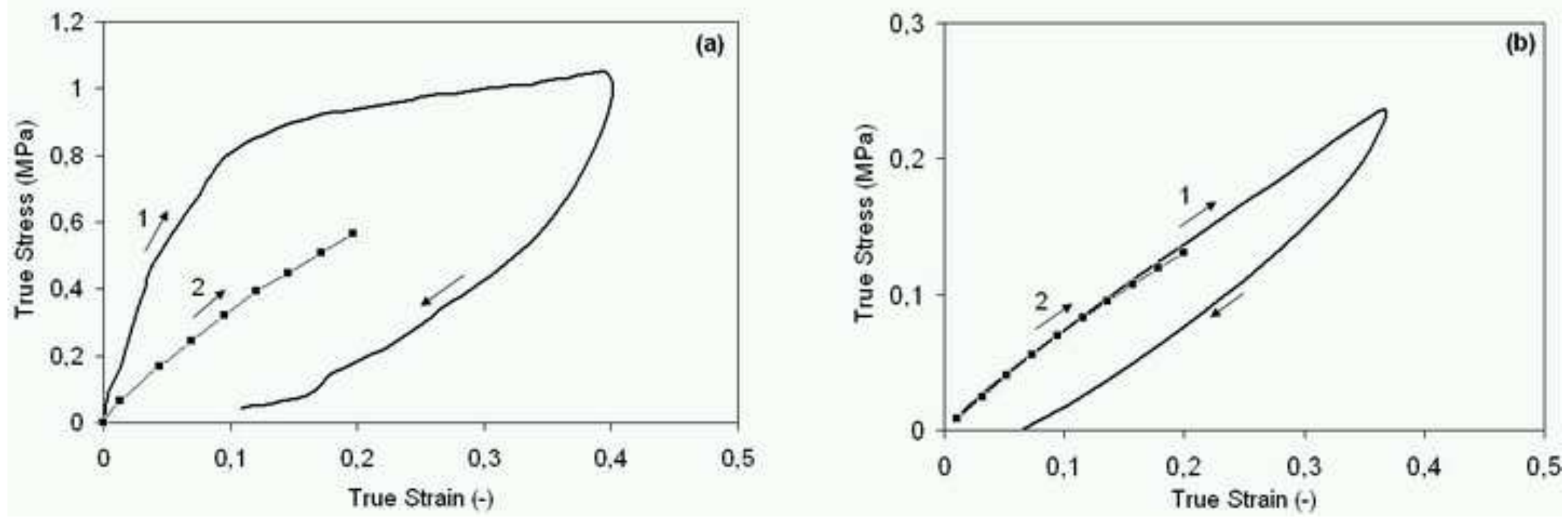
Filler in infinite cluster

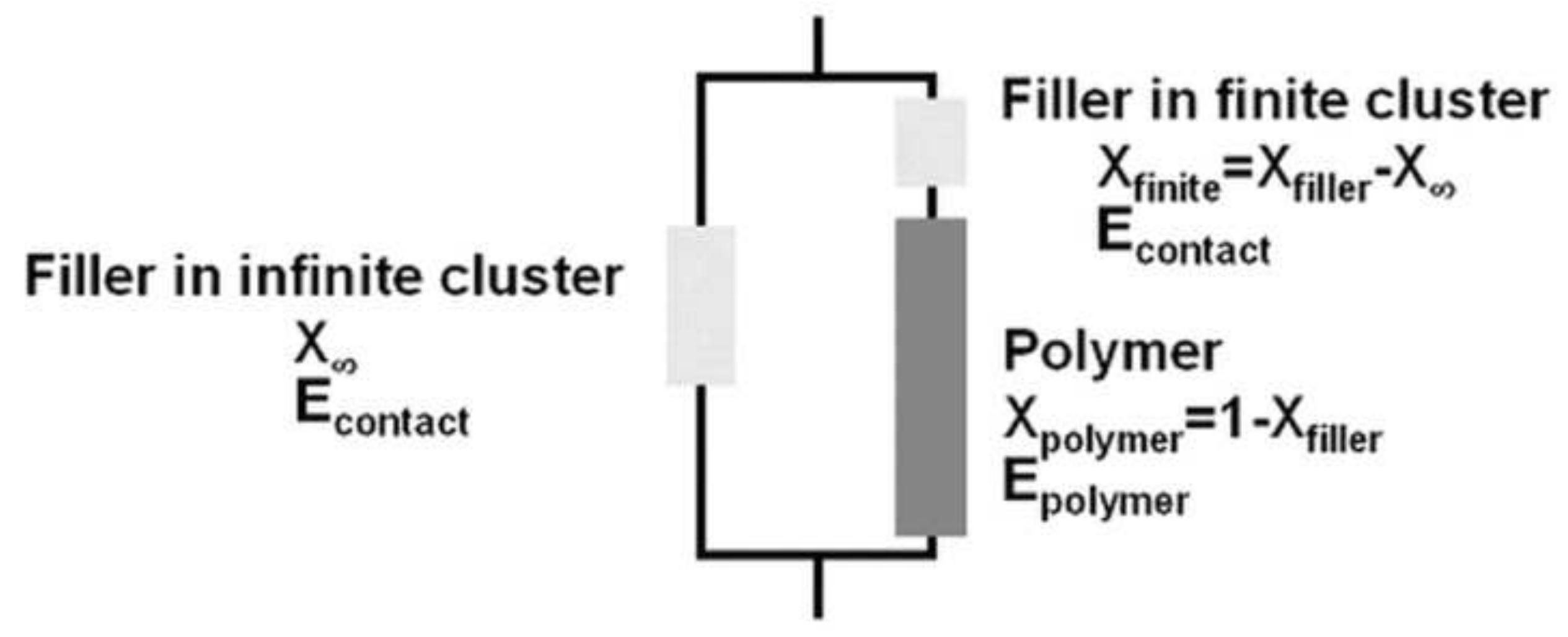

Filler in finite cluster $X_{\text {finite }}=X_{\text {filler }}-X_{\infty}$ $E_{\text {contact }}$

\section{Polymer} $X_{\text {polymer }}=1-X_{\text {filler }}$ $\mathbf{E}_{\text {polymer }}$ 
Figure(s)
Click here to download high resolution image

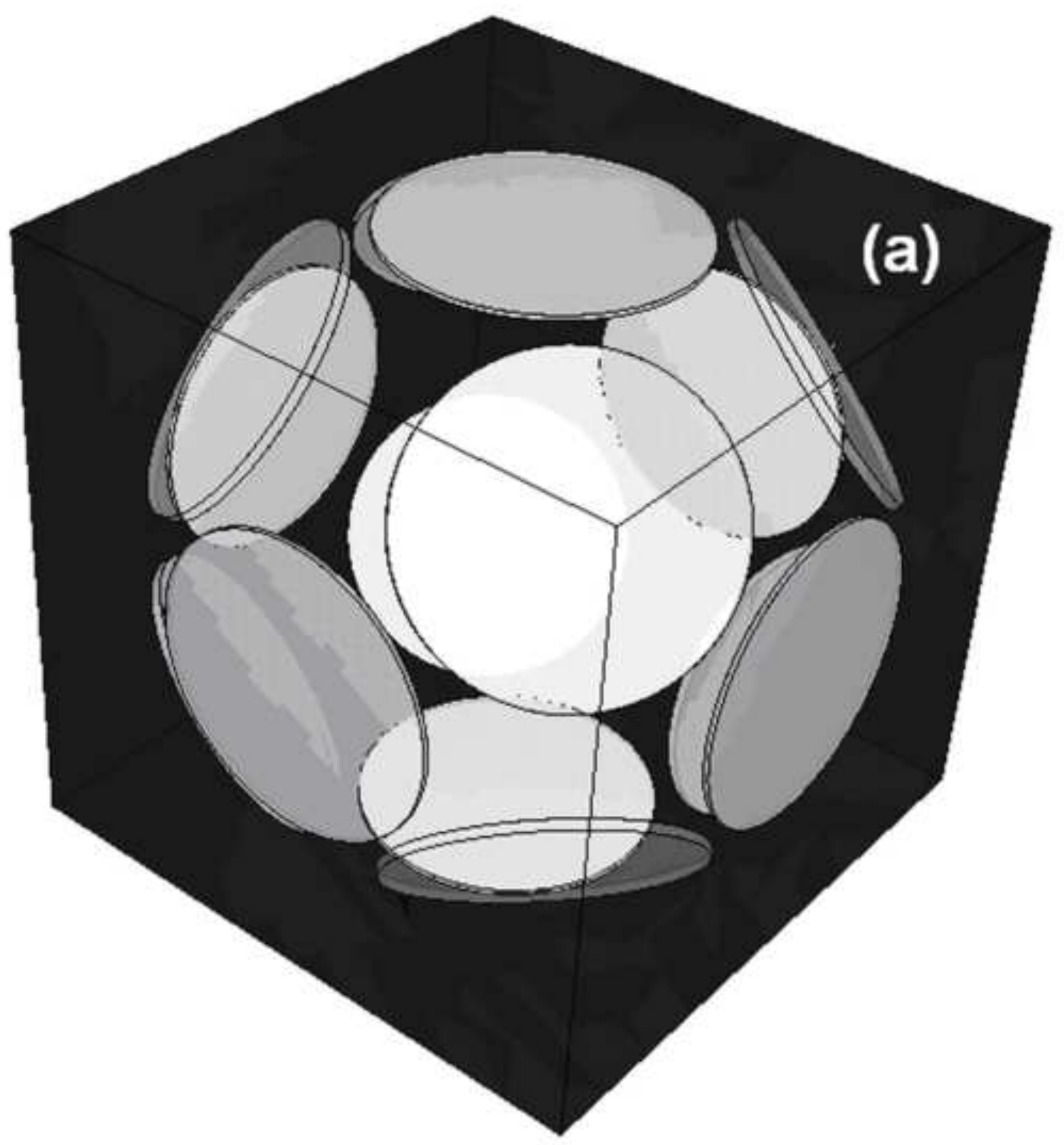


Figure(s)
Click here to download high resolution image

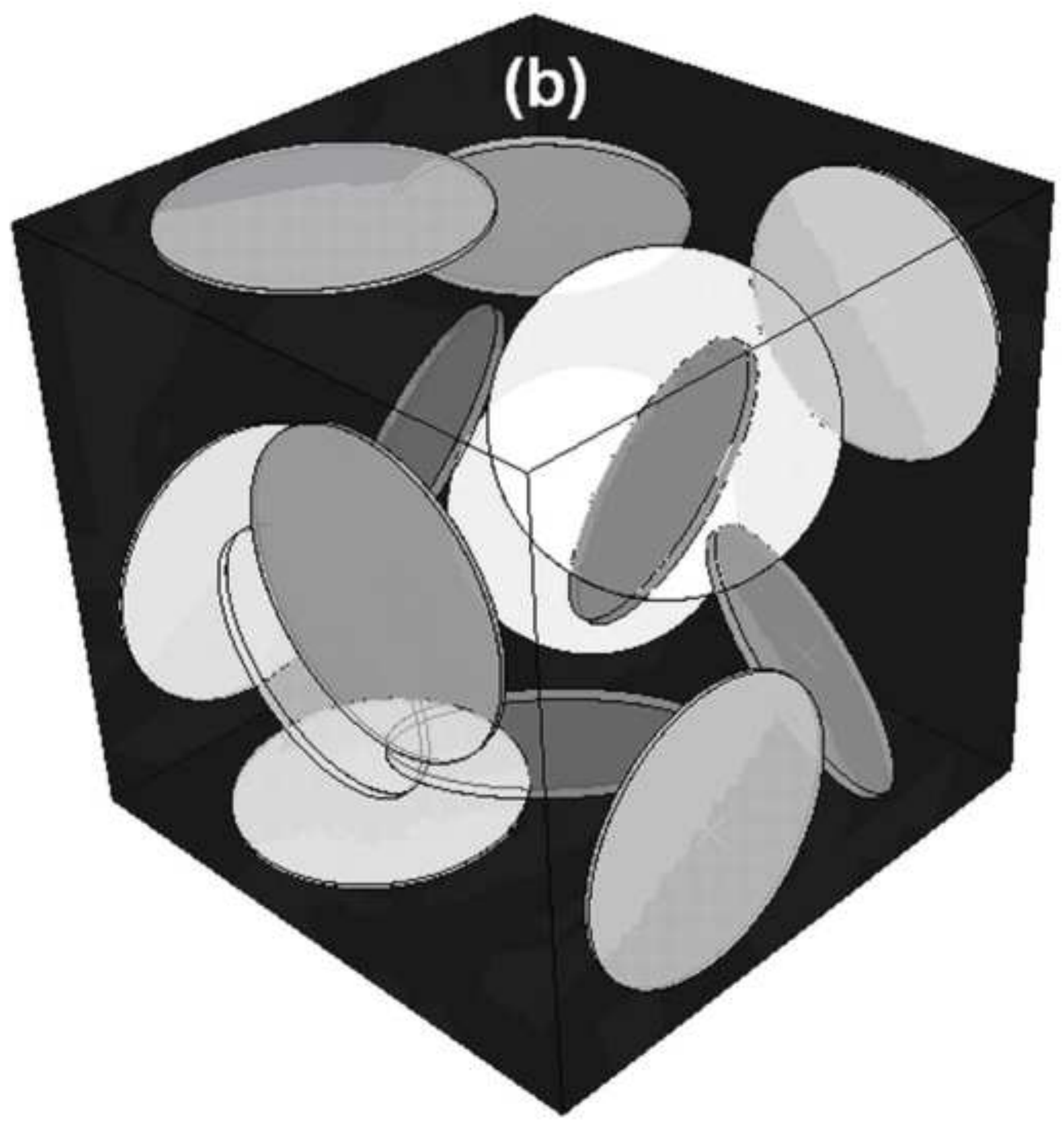




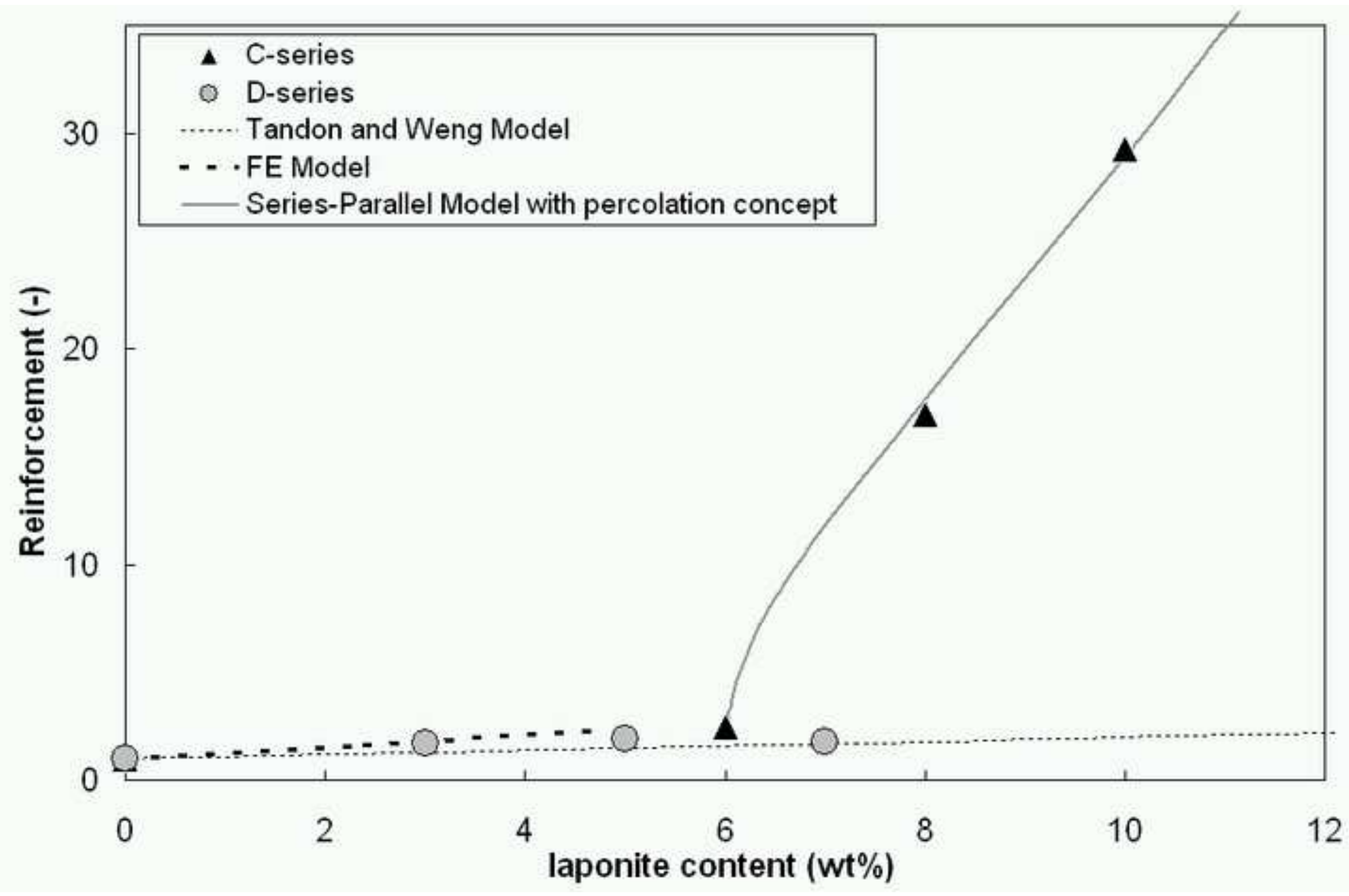




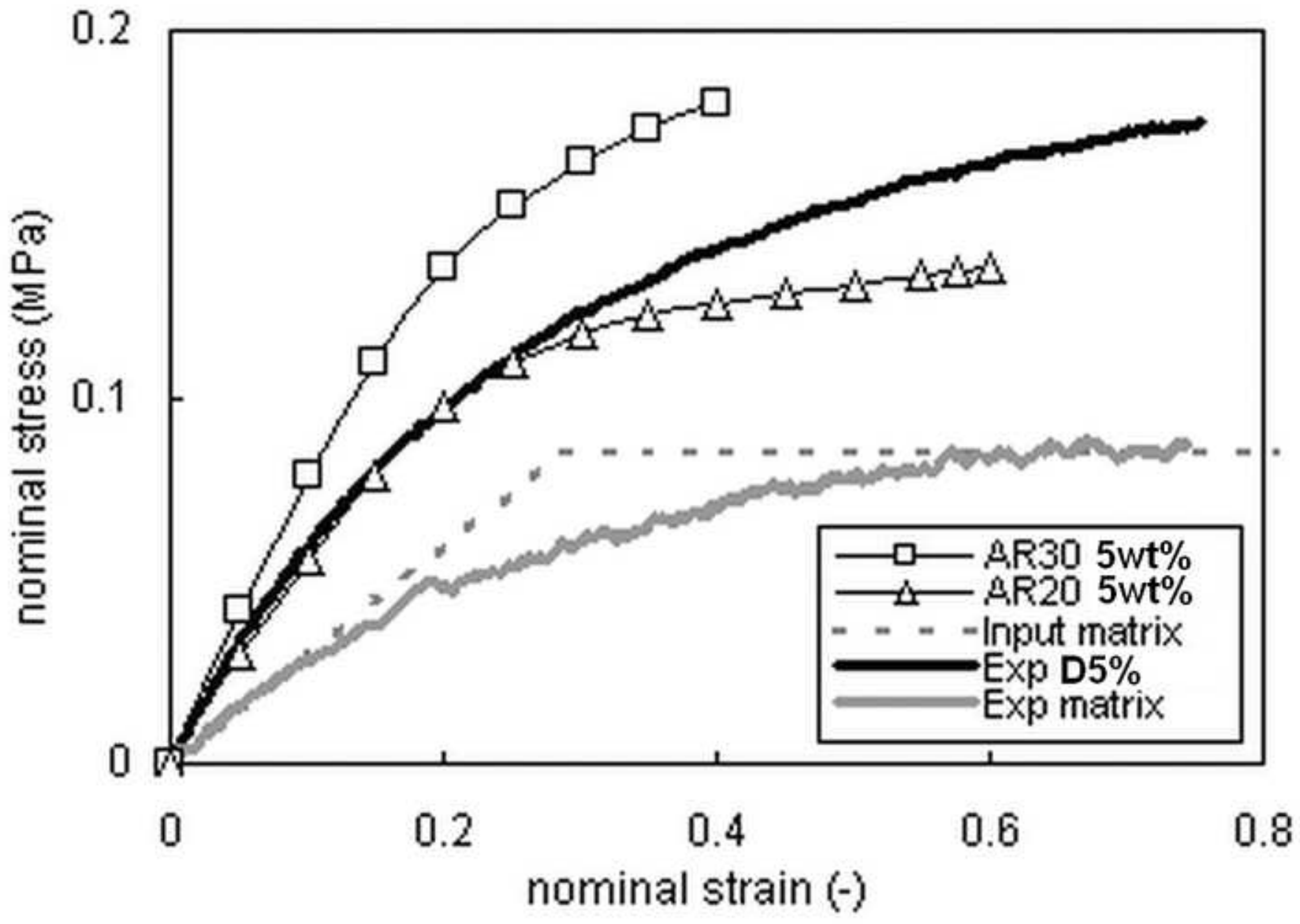


Click here to download high resolution image
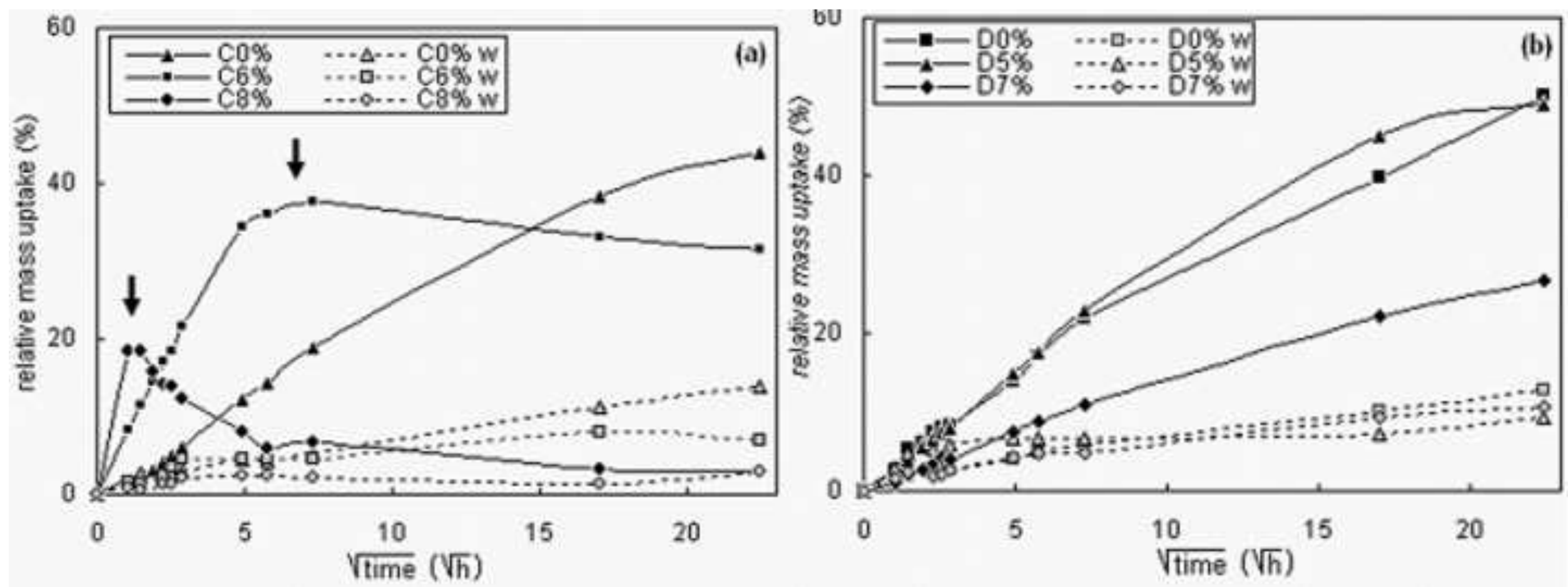
Peptizer on clay Dowfax on latex

\section{Particle}

$D D A B$ on clay

Dowfax on latex

Clay contacts

Path for water diffusion $\rightarrow$ Fast salt dissolution

\section{fresh \\ film}

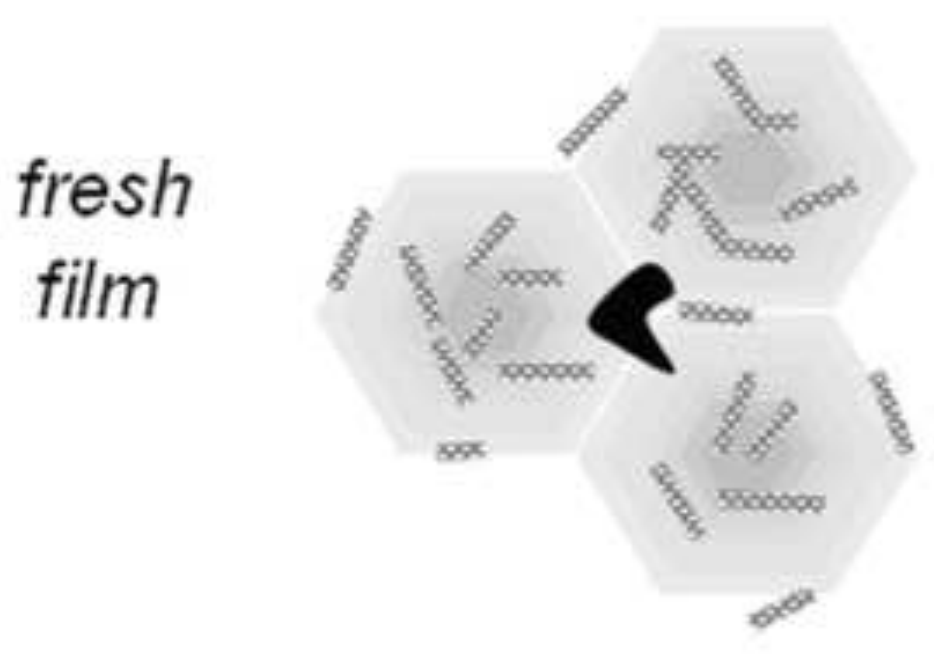
No clay contacts
No path for water diffusion
$\rightarrow$ Slow salt dissolution

(C) sample

(D) sample 


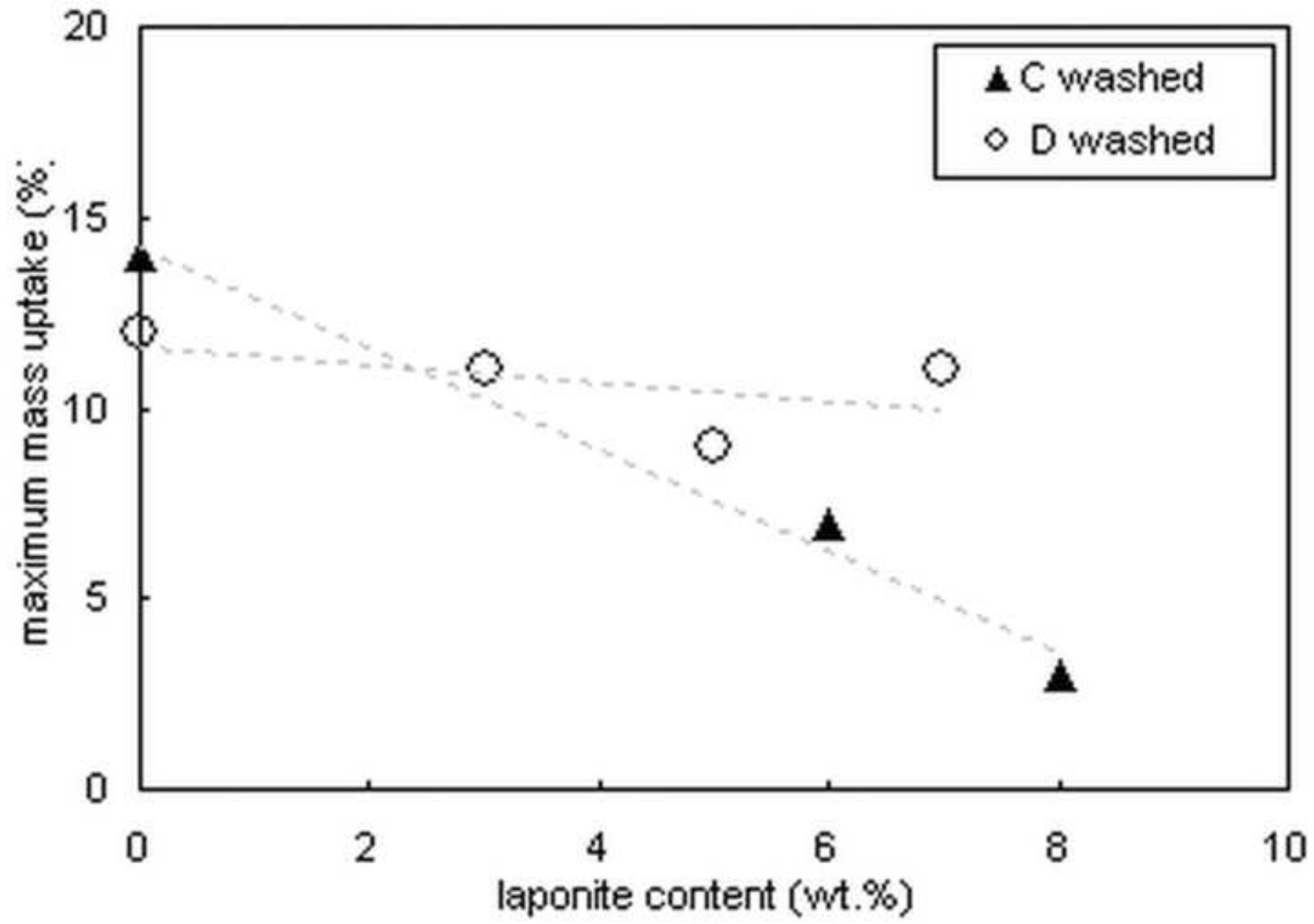


Figure(s)
Click here to download high resolution image

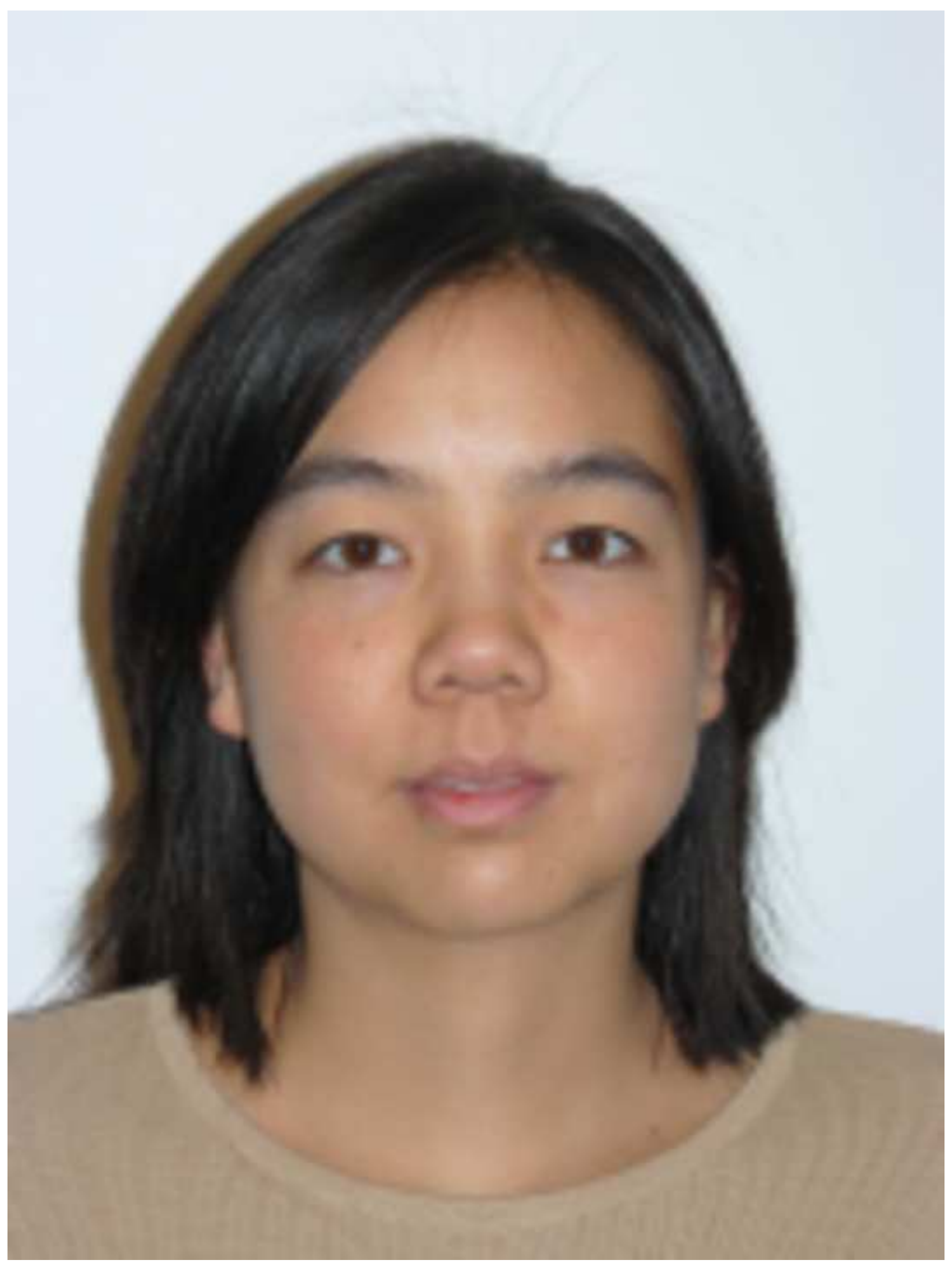


Figure(s)
Click here to download high resolution image

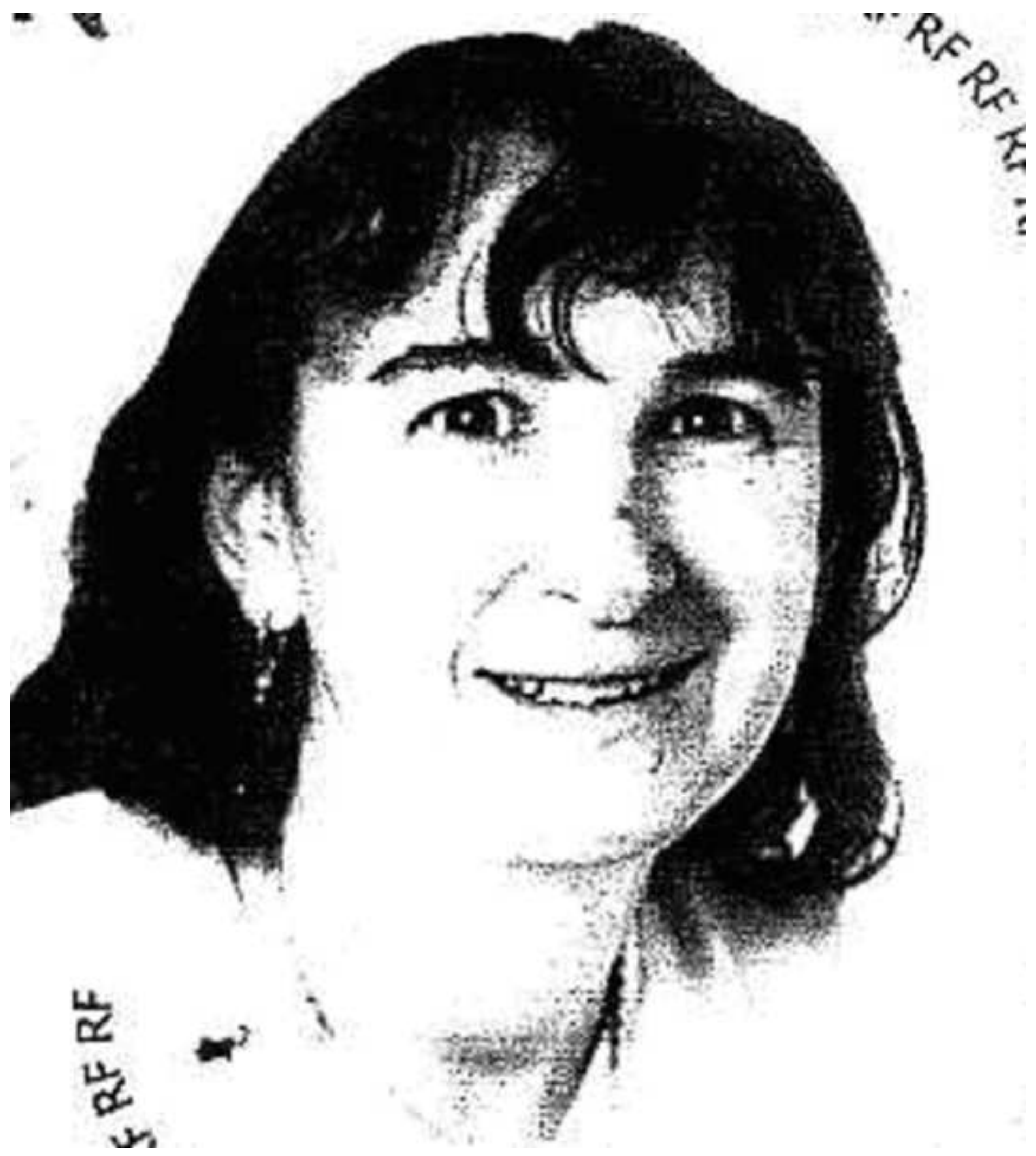


Figure(s)
Click here to download high resolution image

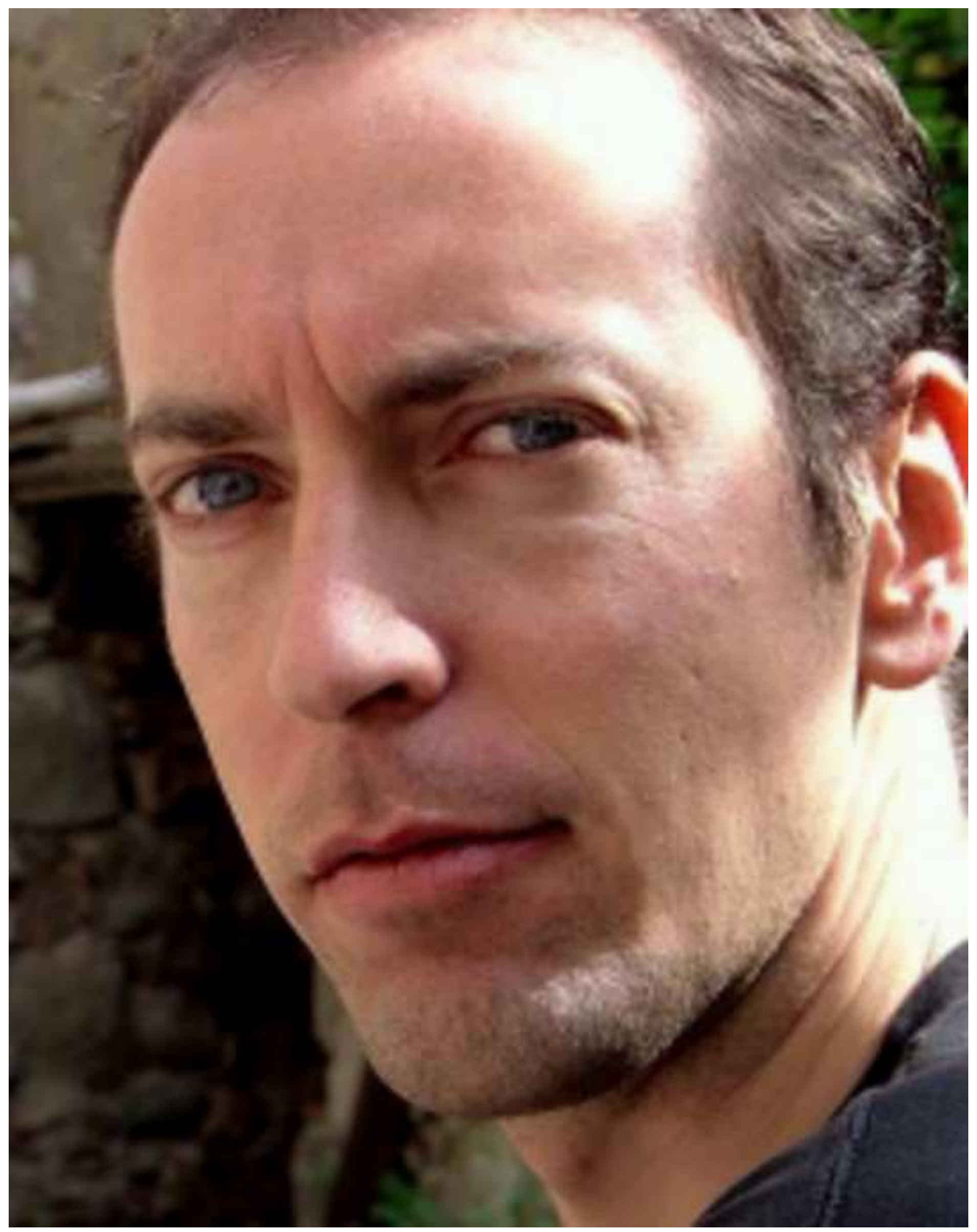


Figure(s)
Click here to download high resolution image

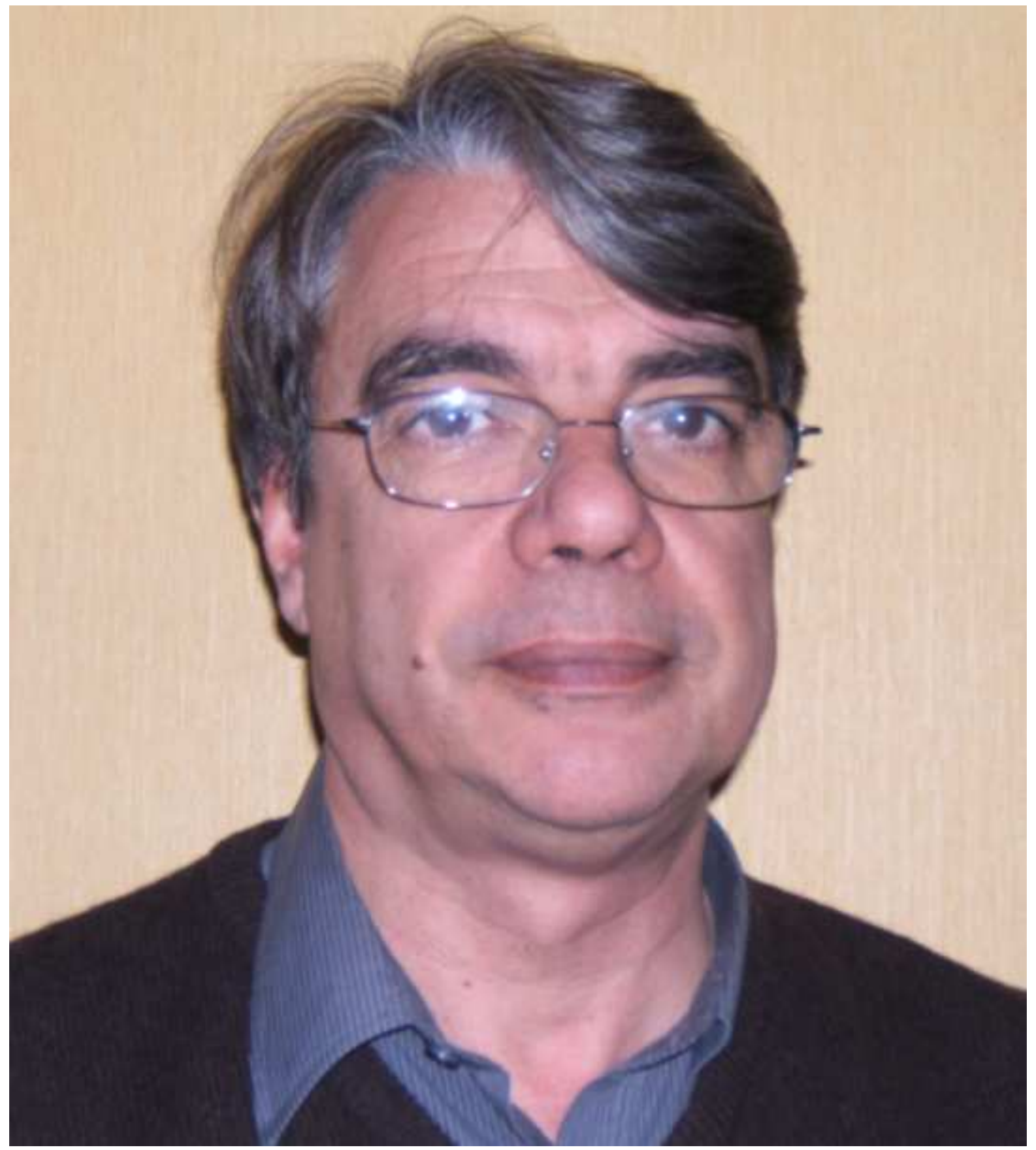


Figure(s)
Click here to download high resolution image

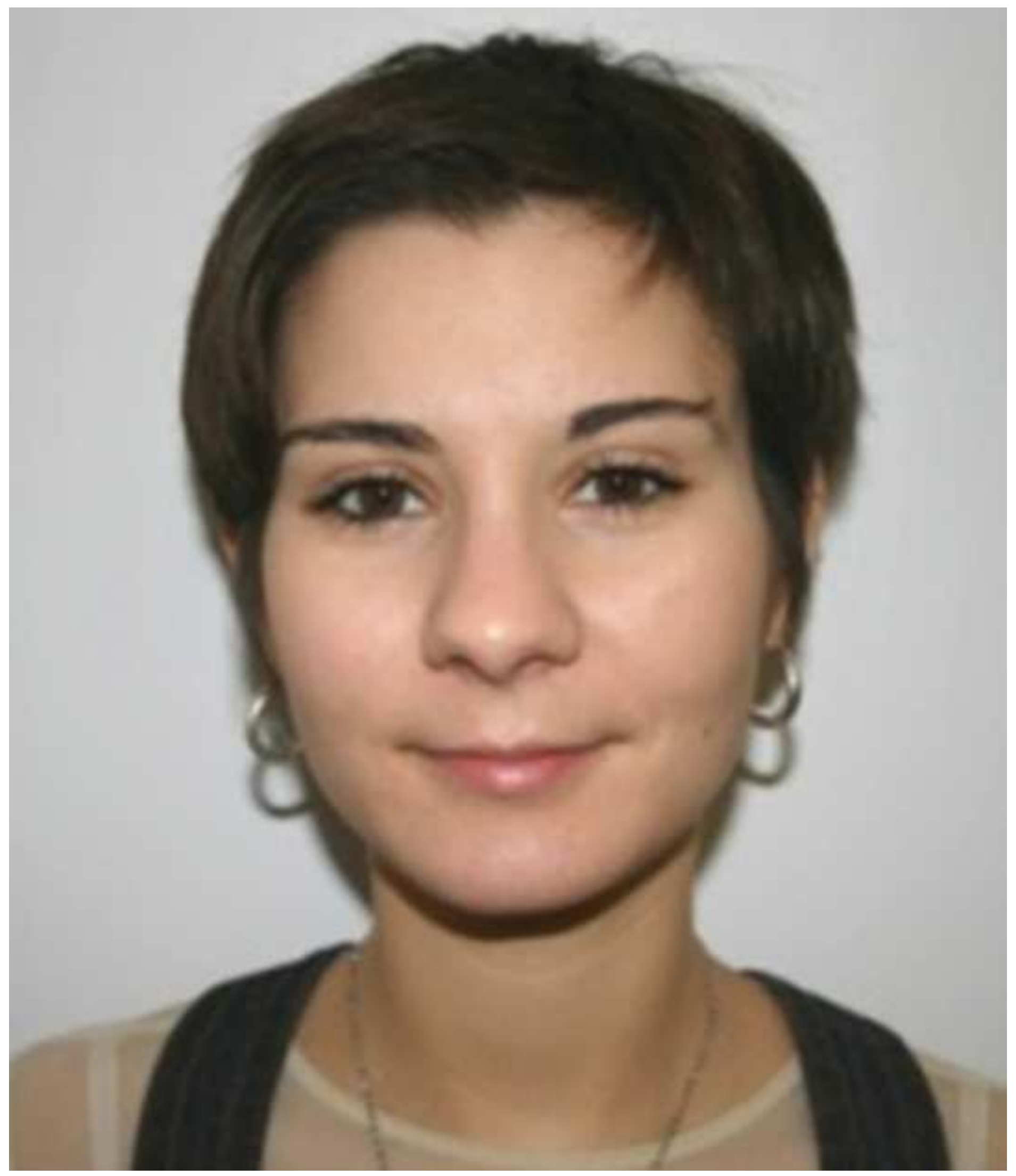


Figure(s)
Click here to download high resolution image

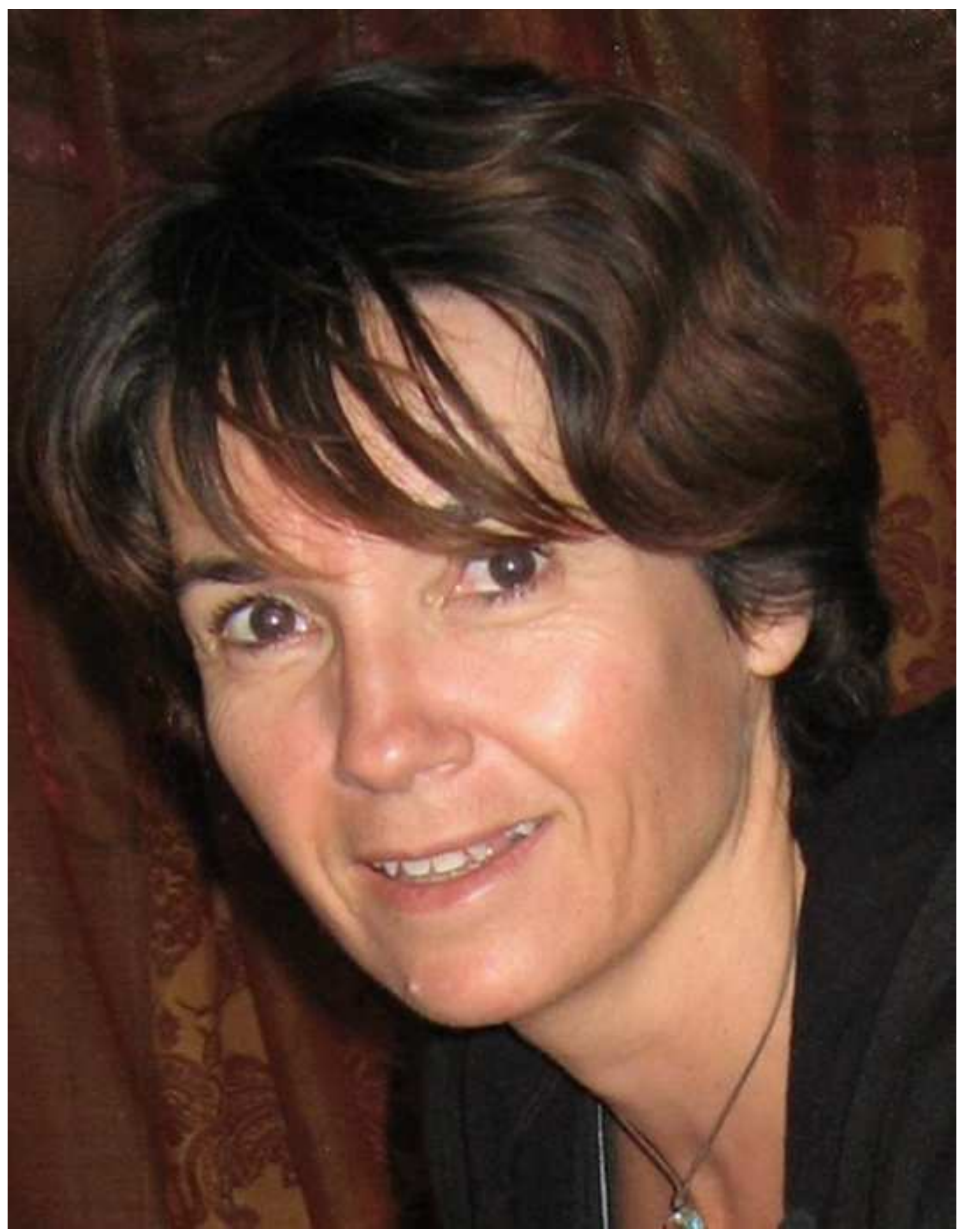


Table 1: sample references obtained by miniemulsion polymerizations of MMA/BA (50/50) by route I (C-series) or by route II (D-series).

\begin{tabular}{ccc|ccc}
\hline Sample & $\begin{array}{c}\text { Laponite } \\
(\mathrm{wt} \%)^{\mathrm{a}}\end{array}$ & Particle $^{\mathrm{b}}$ size (nm) & sample & $\begin{array}{r}\text { Laponite } \\
(\mathrm{wt} \%)^{\mathrm{a}}\end{array}$ & Particle $^{\mathrm{b}}$ size (nm) \\
\hline C 0\% & 0.0 & 68 & D 0\% & 0.0 & 97 \\
$\mathrm{C} 6 \%$ & 5.7 & 97 & D 3\% & 3.4 & 100 \\
$\mathrm{C} 8 \%$ & 8.1 & 83 & D 5\% & 5.1 & 110 \\
$\mathrm{C} 10 \%$ & 10.0 & 81 & D 7\% & 6.5 & 117 \\
\hline
\end{tabular}

${ }^{a}$ Laponite content measured by thermo-gravimetric analysis (ATG) using NETSZCH T209 $\left(30^{\circ} \mathrm{C}\right.$ to $800^{\circ} \mathrm{C}$ under air flow). ${ }^{b}$ Determined by Dynamic Light Scattering (DLS) using a Malvern Autosizer Lo-C.

Table 2: List of "salts" and their content in each sample,

\begin{tabular}{cccccc}
\hline Sample & $\begin{array}{c}\text { Laponite- } \\
\text { Laponite contacts }\end{array}$ & $\begin{array}{c}\text { Dowfax content } \\
(\mathrm{wt} \%)\end{array}$ & $\begin{array}{c}\text { DDAB } \\
(\mathrm{wt} \%)\end{array}$ & $\begin{array}{c}\text { Peptizer } \\
(\mathrm{wt} \%)\end{array}$ & $\begin{array}{c}\text { Salt content } \\
\text { (wt\%) }\end{array}$ \\
\hline C 0\% & - & 1.9 & - & 0.0 & 1.9 \\
C 6\% & Yes & 1.7 & - & 0.4 & 2.1 \\
C 8\% & Yes & 1.7 & - & 0.5 & 2.2 \\
C 10\% & Yes & 1.6 & - & 0.5 & 2.1 \\
D 0\% & - & 1.8 & 0.0 & - & 1.8 \\
D 3\% & No & 1.8 & 0.2 & - & 2.0 \\
D 5\% & No & 1.8 & 0.4 & - & 2.2 \\
D 7\% & No & 1.7 & 0.5 & - & 2.2 \\
\hline
\end{tabular}

